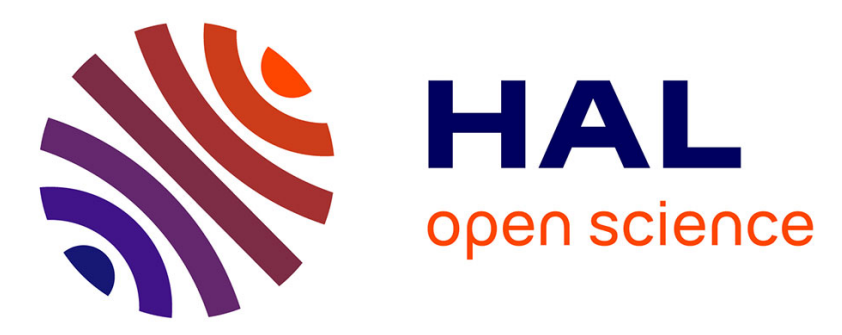

\title{
Absorbing Boundary Conditions for the Two-Dimensional Schrödinger Equation with an Exterior Potential. Part I: Construction and a priori Estimates
}

Xavier Antoine, Christophe Besse, Pauline Klein

\section{- To cite this version:}

Xavier Antoine, Christophe Besse, Pauline Klein. Absorbing Boundary Conditions for the TwoDimensional Schrödinger Equation with an Exterior Potential. Part I: Construction and a priori Estimates. Mathematical Models and Methods in Applied Sciences, 2012, 22 (10), pp.1250025. 10.1142/S021820251250025X . hal-00755639

\author{
HAL Id: hal-00755639 \\ https://hal.science/hal-00755639
}

Submitted on 21 Nov 2012

HAL is a multi-disciplinary open access archive for the deposit and dissemination of scientific research documents, whether they are published or not. The documents may come from teaching and research institutions in France or abroad, or from public or private research centers.
L'archive ouverte pluridisciplinaire HAL, est destinée au dépôt et à la diffusion de documents scientifiques de niveau recherche, publiés ou non, émanant des établissements d'enseignement et de recherche français ou étrangers, des laboratoires publics ou privés. 


\title{
Absorbing boundary conditions for the two-dimensional Schrödinger equation with an exterior potential. Part I: construction and a priori estimates *
}

\author{
Xavier Antoine $^{\dagger} \quad$ Christophe Besse $^{\ddagger} \quad$ Pauline Klein $^{\S}$
}

\begin{abstract}
The aim of this paper is to construct some classes of absorbing boundary conditions for the two-dimensional Schrödinger equation with a time and space varying exterior potential and for general convex smooth boundaries. The construction is based on asymptotics of the inhomogeneous pseudodifferential operators defining the related Dirichlet-to-Neumann operator. Furthermore, $a$ priori estimates are developed for the truncated problems with various increasing order boundary conditions. The effective numerical approximation will be treated in a second paper.
\end{abstract}

\section{Contents}

1 Introduction

2 What we already know and what remains true compared to the one-dimensional case

2.1 The half-space case and a null potential . . . . . . . . . . . . . . . . 4

2.2 The time dependent potential case . . . . . . . . . . . . . . . . . . 4

3 Specific aspects of the two-dimensional case $\quad \mathbf{5}$

3.1 Choice of the boundary and local parameterization . . . . . . . . . . . . 5

3.2 Discussion on the equivalence between the two strategies . . . . . . . . . . . . 6

3.3 Pseudodifferential operator calculus for the $2 \mathrm{D}$ case . . . . . . . . . . . . 7

$3.4 \quad$ E-quasi hyperbolic, elliptic and glancing zones . . . . . . . . . . . . . . . 8

\footnotetext{
*The authors are partially supported by the French ANR fundings under the project MicroWave NT09_460489.

${ }^{\dagger}$ Institut Elie Cartan Nancy, Nancy-Université, CNRS UMR 7502, INRIA CORIDA Team, Boulevard des Aiguillettes B.P. 239, F-54506 Vandoeuvre-lès-Nancy, France (Xavier.Antoine@iecn.u-nancy.fr).

${ }^{\ddagger}$ Laboratoire Paul Painlevé, Université Lille Nord de France, CNRS UMR 8524, INRIA SIMPAF Team, Université Lille 1 Sciences et Technologies, Cité Scientifique, 59655 Villeneuve d'Ascq Cedex, France. (Christophe.Besse@math.univ-lille1.fr).

$\S$ Laboratoire de Mathématiques de Besançon, CNRS UMR 6623, Université de Franche-Comté, 16 route de Gray, 25030 Besançon, France. (Pauline.Klein@iecn.u-nancy.fr).
} 
4 Two possible strategies $\quad 9$

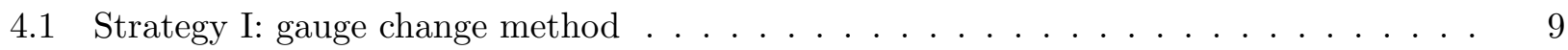

4.2 Strategy II: direct method . . . . . . . . . . . . . . . . . . . . . . 10

4.3 Unification of the strategies . . . . . . . . . . . . . . . . . . . 10

4.4 Symbolic system . . . . . . . . . . . . . . . . . . . . . 10

4.5 Adding terms in the principal symbol . . . . . . . . . . . . . . . 13

5 Strategy I: gauge change $\quad 14$

5.1 Choice of the principal symbol _. . . . . . . . . . . . . . . . . . . 14

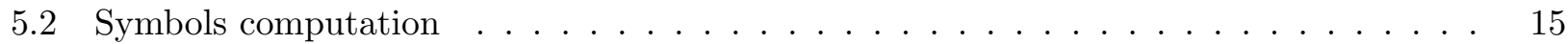

5.3 Interpretation of the ABCs for the Taylor approach . . . . . . . . . . . . . . . 16

5.4 Interpretation of the ABCs for the Padé approach . . . . . . . . . . . . . 19

6 Strategy II: direct method $\quad 20$

6.1 Computation of the symbols . . . . . . . . . . . . . . . . . . 21

6.2 Interpretation of the ABCs for the Taylor approach . . . . . . . . . . . . . . 22

6.3 Interpretation of the ABCs and Padé approach . . . . . . . . . . . . . . 23

$\begin{array}{llr} & \text { A priori estimates } & \mathbf{2 6}\end{array}$

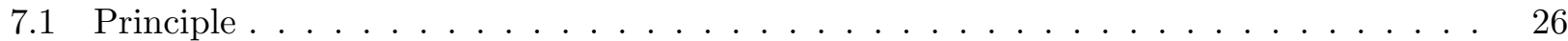

$7.2 \quad$ A priori estimates for $\mathrm{ABC}_{2, T}^{M} \ldots \ldots \ldots \ldots \ldots \ldots \ldots \ldots$

$7.3 \quad$ A priori estimates for $\mathrm{ABC}_{1, T}^{M} \ldots \ldots \ldots \ldots \ldots \ldots \ldots \ldots$

8 Conclusion $\quad 33$

\section{Introduction}

The aim of this paper is to propose some Absorbing Boundary Conditions (ABCs) for the twodimensional linear time-dependent Schrödinger equation [1] with a potential $V$

$$
\left\{\begin{array}{l}
i \partial_{t} u+\Delta u+V(x, y, t) u=0, \quad(x, y) \in \mathbb{R}^{2}, t>0 \\
u(x, y, 0)=u_{0}(x, y), \quad(x, y) \in \mathbb{R}^{2}
\end{array}\right.
$$

where $u_{0} \in L^{2}\left(\mathbb{R}^{2}\right)$ is compactly supported in the future bounded spatial computational domain $\Omega$, with fictitious boundary $\Sigma$. The potential function is $C^{\infty}$, space and time dependent and real-valued. We assume that the smoothness of $V$ is at least satisfied outside $\left.\Sigma_{T}:=\Sigma \times\right] 0 ; T$ [, $T$ being the final time of computation. The computational domain is $\left.\Omega_{T}=\Omega \times\right] 0 ; T$. Under suitable conditions, the initial boundary value problem $(1)$ is well-posed $[12,13]$. Moreover, in the free-space the $L^{2}$-norm of the solution is conserved

$$
\forall t>0, \quad\|u(t)\|_{L^{2}\left(\mathbb{R}^{2}\right)}^{2}=\int_{\mathbb{R}^{2}}|u(x, y, t)|^{2} d x d y=\left\|u_{0}\right\|_{L^{2}\left(\mathbb{R}^{2}\right)}^{2}
$$

where $\|\cdot\|_{L^{2}\left(\mathbb{R}^{2}\right)}^{2}$ is the $L^{2}\left(\mathbb{R}^{2}\right)$-norm. Finally, $\mathbf{n}$ is the outwardly directed unit normal vector to $\Sigma$. 
The main difficulty here in designing ABCs is that we include a general potential $V:=V(x, y, t)$. In practical applications, being able to handle correctly potential effects is an important, active and not completely understood topic in physics $[23,15,14,21,22]$. Here, we assume that the potential makes the wave outgoing to the bounded domain $\Omega$. In the one-dimensional case, a sufficient condition is that $V$ is an acceleration or a repulsive potential [5]. Examples of potentials which cannot be considered are the confining potentials. In the two-dimensional case, a definition of admissible (repulsive) potential does not exist. For this reason, we assume that the outgoing wave assumption holds without giving a mathematical definition. This point is probably interesting and important to discuss but also out of the scope of the paper. Our contribution focuses on the effective construction of ABCs and some of their properties. Their discretization and numerical comparison will be included in a companion paper [6].

In the one-dimensional case, $\mathrm{ABC}$ for some special time independent potentials can be obtained via explicit calculations of the Dirichlet-to-Neumann (DtN) operator by using special functions (for example Airy's functions) $[20,19]$ or particular techniques (Floquet's theory for sinusoidal potentials [29]). Handling general potentials can be achieved by using pseudodifferential operator theory and symbolic asymptotic expansions of the DtN map. In [5], we introduced two strategies to build robust and accurate ABCs making use of the Engquist \& Majda [16, 17] approach adapted to the Schrödinger equation $[5,7,3,1,8,25,26,18,11]$. In the present paper, we develop the extension of the theory to the two-dimensional case for general smooth convex boundaries $\Sigma$. As we will see, the two strategies of the one-dimensional case can be extended to the two-dimensional case. However, since the principal symbol of the DtN operator is more complicated, further (high frequency) asymptotic expansions are required and lead in fact to four families of $\mathrm{ABCs}$, two for each strategy. After designing these ABCs, we also investigate the obtention of a priori estimates, which from the semi discrete point of view would correspond to prove stability of the discretization schemes. Let us note that the technique of Perfectly Matched Layer (PML) [10] can also be used to bound the computational domain for spatially dependent potentials. We refer to [28] for more details.

The paper is structured as follows. In the second Section, we review some results known for special two-dimensional problems and explain what can be extended from the one- to the two-dimensional case. In Section 3, we introduce a local parameterization of the boundary and discuss the possible links between the two strategies. Next, specific pseudodifferential operators results are summarized and the $E$-quasi hyperbolic, elliptic and glancing zones are defined. In Section 4, we explain in details the two strategies. The first one (strategy I) is called the gauge change and the second one (strategy II) is the direct method. We unify the notations and then develop the symbolic system which gives the asymptotic expansion of the total symbol of the DtN map. We then discuss the choice of the principal symbol of the DtN map which plays a key role in the accuracy of the ABCs. Section 5 deals with the gauge change strategy (strategy I) where we detail the calculations and the construction of ABCs. Section 6 does the same developments but for the direct strategy (strategy II). We state $a$ priori estimates in Section 7 and conclude in Section 8. 


\section{What we already know and what remains true compared to the one-dimensional case}

\subsection{The half-space case and a null potential}

Let us consider the positive half-space $\{x>0\}$. We set $\Omega:=\{x<0\}$ with boundary $\Sigma=\{x=0\}$. Let us assume that the potential $V$ is equal to zero in the right half-space. Then we can prove that the (exact) Transparent Boundary Condition (TBC) for the problem

$$
i \partial_{t} u+\partial_{x}^{2} u+\partial_{y}^{2} u+V u=0, \quad(x, y) \in \mathbb{R}^{2}, t>0,
$$

is given by the Dirichlet-to-Neumann (DtN) map

$$
\partial_{\mathbf{n}} u-i \sqrt{i \partial_{t}+\Delta_{\Sigma}} u=0, \quad(x, y) \in \Sigma_{T}
$$

This TBC can be obtained by Fourier transform in time $t$ and space $y$. Then, the resulting differential equation in $x$ is solved explicitly leading to the linear combination of two traveling waves. It is next sufficient to kill the incoming wave and to derive according to $x$ (normal derivative $\partial_{\mathbf{n}}:=\partial_{x}$ ) to get (3) in the $(y, t)$ Fourier space. An inverse Fourier transform then gives the result. In our case, the Laplace-Beltrami operator $\Delta_{\Sigma}$ is $\partial_{y}^{2}$. The transparent operator is given by a square-root Schrödinger operator on the boundary $\Sigma$. This operator is nonlocal in time and space. We will see later that this operator can be localized (for example by a Taylor expansion) or globally localized in time and space (through rational approximants $[6]$ ).

\subsection{The time dependent potential case}

In the one-dimensional case and when the potential is only time dependent $V(x, t)=V(t)$ outside $\Omega$, a gauge change leads to work with a free potential Schrödinger equation. If $u$ is solution to: $i \partial_{t} u+\partial_{x}^{2} u+V(t) u=0$, and $v$ is defined by

$$
v(x, t)=u(x, t) e^{-i \int_{0}^{t} V(s) d s},
$$

then $v$ is solution to: $i \partial_{t} v+\partial_{x}^{2} v=0$, for which the TBC is known. In the two-dimensional case, this property remains true. If $V(x, y, t)=V(t)$ and $u$ is solution to

$$
i \partial_{t} u+\partial_{x}^{2} u+\partial_{y}^{2} u+V(t) u=0
$$

then, by defining

$$
v(x, y, t)=u(x, y, t) e^{-i \int_{0}^{t} V(s) d s},
$$

$v$ is solution to the free potential equation: $i \partial_{t} v+\partial_{x}^{2} v+\partial_{y}^{2} v=0$. This result is independent of the coordinates system $(x, y)$. 


\section{Specific aspects of the two-dimensional case}

\subsection{Choice of the boundary and local parameterization}

Let us consider a rectangular domain $\Omega$ parameterized in cartesian coordinates $(x, y)$ with corners $\left(x_{1}, y_{1}\right),\left(x_{1}, y_{2}\right),\left(x_{2}, y_{2}\right)$ and $\left(x_{2}, y_{1}\right)$. For the vertical right boundary $\Sigma_{r}=\left\{(x, y) \in \mathbb{R}^{2} / x=x_{2}, y \in\right.$ $\left.\left[y_{1} ; y_{2}\right]\right\}$, we use the pseudodifferential operator calculus associated with the partial Fourier transform in $(y, t)$ to get the DtN operator according to $\partial_{x}$ by considering the right half-space. Then, we obtain a TBC on $\Sigma_{d}$. The problem is that this boundary condition is singular at $\left(x_{2}, y_{2}\right)$ and does not smoothly match with the boundary condition that one would get on $\Sigma_{h}:=\left[x_{1} ; x_{2}\right] \times\left\{y_{2}\right\}$ by considering a partial Fourier transform in $(x, t)$. This means that additional continuity conditions at the corners must be developed (see e.g. [27] for the wave equation). To the best of our knowledge, this question has not been treated yet for the Schrödinger equation. Furthermore, to have an outgoing solution to the computational domain $\Omega$, a convex (regular) boundary $\Sigma$ must be considered. Therefore, we try to write a boundary condition on a smooth (closed) convex boundary $\Sigma$ parameterized by its curvilinear abscissa $s \in[a ; b]$. The assumption about the convexity implies that the local curvature at $s, \kappa=\kappa(s)$, is positive.

Let $\Omega$ be a convex domain with smooth boundary $\Sigma$. We do not detail here all the calculations and refer to [3] for more explanations concerning the change of variables. For a point $M$ of $\Sigma$ with coordinates $(x, y)$, we designate by $\boldsymbol{\tau}$ the tangential vector to $\Sigma$ at $M$ and $\mathbf{n}$ the outwardly directed unit normal vector. In the local coordinates system associated with $M$, a point $M^{\prime}$ close to the boundary is connected to its coordinates $r$ and $s$. Since $\Omega$ is convex, the projection of the point $M^{\prime}$ onto the boundary $\Sigma$ is unique, giving hence its curvilinear abscissa $s$. The radial coordinate $r$ is the distance from point $M^{\prime}$ to its projection according to the outgoing unitary normal vector. Hence, $\Sigma$ can be denoted by $\Sigma_{0}$, if $\Sigma_{r}$ designates the parallel surface to $\Sigma$ at distance $r$. Since $\Sigma$ is convex, we can restrict ourselves to positive values of $r$, bounded from above by a small parameter $\varepsilon$, and so $r \in[0 ; \varepsilon]$. The laplacian in local coordinates $(r, s)$ writes down $[4,2]$

$$
\Delta_{r}=\partial_{r}^{2}+\kappa_{r} \partial_{r}+h^{-1} \partial_{s}\left(h^{-1} \partial_{s}\right)
$$

with the scaling factor $h: h=1+r \kappa$ and $\kappa_{r}$ the curvature at $M^{\prime}$ on the parallel surface $\Sigma_{r}: \kappa_{r}=h^{-1} \kappa$. For the sake of conciseness, we denote by $\tilde{u}$ the function $u$ written in the local system

$$
u(x, y, t)=\tilde{u}(r, s, t), \quad(x, y) \in \mathbb{R}^{2},(r, s) \in[0 ; \varepsilon] \times[a ; b], t>0,
$$

and $V_{r}$ the locally rewritten potential function

$$
V(x, y, t)=V_{r}(r, s, t), \quad(x, y) \in \mathbb{R}^{2},(r, s) \in[0 ; \varepsilon] \times[a ; b], t>0 .
$$

The Schrödinger equation for system (1) is then

$$
\left.\left.i \partial_{t} \tilde{u}+\partial_{r}^{2} \tilde{u}+\kappa_{r} \partial_{r} \tilde{u}+h^{-1} \partial_{s}\left(h^{-1} \partial_{s}\right) \tilde{u}+V_{r} \tilde{u}=0, \quad(r, s, t) \in[0 ; \varepsilon] \times[a ; b] \times\right] 0 ; T\right],
$$

where $r$ and $s$ parameterize the domain $\Omega$ and $t>0$. In the sequel, we identify $u$ to $\tilde{u}$. 


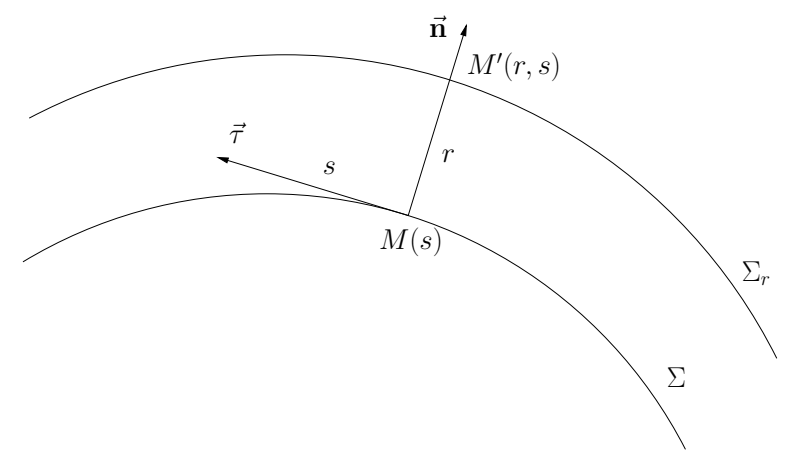

Figure 1: Local generalized coordinates system.

\subsection{Discussion on the equivalence between the two strategies}

In the one-dimensional case, when $V$ is only $x$ dependent and by applying a partial Fourier transform, we have the equivalence between the two (gauge change and direct) strategies according to the identity (see Lemma 2, page 320, in [5])

$$
O p(a(\tau-V(x))) u=e^{i t V} O p(a(\tau))\left(e^{-i t V} u(x, t)\right)
$$

In two dimensions, if one considers the solution to the Schrödinger equation in the half-space $\{x>0\}$, we apply a Fourier transform with respect to $y$ and $t$. To have a similar property, we have to assume that $V$ only depends on one spatial variable $V(x, y, t)=V(x)$, which is unrealistic for practical applications.

If one works in the local coordinates system with $r$ and $s$, it seems reasonable to consider the dependence properties according to the computational domain geometry. For example, a radial variable in a circular domain is characterized by a null tangential variation. In the local system, the potential writes down $V(r, s, t)$ and we work with the Fourier transform in $s$ and $t$. To get an equivalence property, we would have to assume that $V$ is $s$ and $t$ independent, which means that the potential is symmetrically radial and time independent and can be considered as quite realistic now. Then, we have the following result.

Lemma 1. If $a$ is a symbol in $S^{m}$, independent of $t$ and $s$, and $V(r, s, t)=V(r)$, then we have the equality

$$
O p(a(\tau-V(r), \xi)) u(r, s, t)=e^{i t V} O p(a(\tau, \xi))\left(e^{-i t V} u(r, s, t)\right),
$$

where $\xi$ designates the Fourier covariable with respect to $s$.

Proof. By definition, the pseudodifferential operator associated with the symbol $a(\tau-V(r), \xi)$ is given by a partial inverse Fourier transform

$$
\begin{aligned}
O p(a(\tau-V(r), \xi)) u & =\mathscr{F}_{(s, t)}^{-1}(a(\tau-V(r), \xi) \hat{u}(r, \xi, \tau)) \\
& =\int_{\mathbb{R}} \int_{\mathbb{R}} a(\tau-V(r), \xi)\left(\mathscr{F}_{(s, t)} u\right)(r, \xi, \tau) e^{i t \tau} e^{i s \xi} d \tau d \xi .
\end{aligned}
$$


By using the following time variable change $\rho=\tau-V(r)$ one gets

$$
O p(a(\tau-V(r), \xi)) u=\int_{\mathbb{R}} \int_{\mathbb{R}} a(\rho, \xi)\left(\mathscr{F}_{(s, t)} u\right)(r, \xi, \rho+V(r)) e^{i t \rho} e^{i t V(r)} e^{i s \xi} d \rho d \xi .
$$

From the translation properties of the Fourier transform we can simplify the expression

$$
O p(a(\tau-V(r), \xi)) u=e^{i t V(r)} \int_{\mathbb{R}} \int_{\mathbb{R}} a(\rho, \xi) \mathscr{F}_{(s, t)}\left((r, s, t) \mapsto e^{-i t V(r)} u(r, s, t)\right)(r, \xi, \rho) e^{i t \rho} e^{i s \xi} d \rho d \xi .
$$

Then, we identify the operator with symbol $a(\rho, \xi)$ applied to the function $(r, s, t) \mapsto e^{-i t V(r)} u(r, s, t)$, i.e.

$$
O p(a(\tau-V(r), \xi)) u=e^{i t V(r)} O p(a(\tau), \xi)\left(e^{-i t V(r)} u(r, s, t)\right),
$$

which proves (9).

\subsection{Pseudodifferential operators for the two-dimensional case and associated sym- bolic calculus}

The functions that we consider in this chapter depend not only on two variables, the local spatial coordinates $r$ and $s$, but also on the time $t$. In this framework, the two-dimensional pseudodifferential operator calculus is realized through the partial Fourier transform $(s, t)$ of a function $f(r, s, t)$. We denote by $\xi$ (respectively $\tau$ ) the covariable of $s$ (respectively $t$ ). We have

$$
\mathscr{F}_{(s, t)}(f(r, s, t))(r, \xi, \tau)=\frac{1}{4 \pi^{2}} \int_{\mathbb{R}} \int_{\mathbb{R}} f(r, s, t) e^{-i t \tau} e^{-i s \xi} d t d s
$$

and we set $\mathscr{F}=\mathscr{F}_{(s, t)}$ in this section. A pseudodifferential operator $P\left(r, s, t, \partial_{s}, \partial_{t}\right)$ with symbol $p(r, s, t, \xi, \tau)$ is defined by

$$
P\left(r, s, t, \partial_{s}, \partial_{t}\right) u(r, s, t)=\mathscr{F}_{(s, t)}^{-1}(p(r, s, t, \xi, \tau) \hat{u}(r, \xi, \tau)),
$$

that is

$$
P\left(r, s, t, \partial_{s}, \partial_{t}\right) u(r, s, t)=\int_{\mathbb{R}} \int_{\mathbb{R}} p(r, s, t, \xi, \tau) \hat{u}(r, \xi, \tau) e^{i t \tau} e^{i s \xi} d \tau d \xi
$$

where $\hat{u}=\mathscr{F} u$.

The inhomogeneous pseudodifferential operator calculus that we use in the paper was introduced in [18] and applied e.g. in [3]. For the sake of conciseness, we only give the useful material needed here. Let $m$ be a real number and $\mathcal{O}$ an open subset of $\mathbb{R}^{2}$. Then (see in [24]), the symbol class $S^{m}\left(\mathcal{O} \times \mathbb{R}^{+}\right)$denotes the linear space of $\mathcal{C}^{\infty}$ functions $a(r, s, t, \tau, \xi)$ in $\mathcal{O} \times \mathbb{R}^{+} \times \mathbb{R}^{2}$ such that for each $K \subseteq \mathcal{O}$ and for all integer indices $k, \alpha_{r}, \alpha_{s}, l$ and $\beta$, there exists a constant $C_{k, \alpha_{r}, \alpha_{s}, l, \beta}(K)$ such that

$$
\left|\partial_{t}^{k} \partial_{r}^{\alpha_{r}} \partial_{s}^{\alpha_{s}} \partial_{\tau}^{l} \partial_{\xi}^{\beta} a(r, s, t, \tau, \xi)\right| \leq C_{k, \alpha_{r}, \alpha_{s}, l, \beta}(K)\left(1+\tau^{2}+\xi^{4}\right)^{m-\beta-2}
$$

for all $(r, s) \in K, t \in \mathbb{R}^{+}$and $(\tau, \xi) \in \mathbb{R}^{2}$.

Let $E=(1,2)$. The homogeneity of a pseudodifferential operator can be deduced from the homogeneity of its symbol with respect to $\left(\xi^{2}, \tau\right)$. Therefore, $\xi^{2}$ and $\tau$ are considered as homogeneous $[18,11]$. This leads to the following definition. 
Definition 1. A function $f(r, s, t, \xi, \tau)$ is said to be E-quasi homogeneous of order $m$ if and only if for all $\mu>0$ and for large $\left(\xi^{2}, \tau\right)$ we have

$$
f\left(r, s, t, \mu \xi, \mu^{2} \tau\right)=\mu^{m} f(r, s, t, \xi, \tau) .
$$

For example, the operator with symbol $\lambda=\sqrt{-\tau-\xi^{2}}$ is first order $E$-quasi homogeneous (with respect to $\left.\left(\xi^{2}, \tau\right)\right)$.

From now on, a $E$-quasi homogeneous pseudodifferential operator of order $m \in \mathbb{Z}$, denoted by $A \in \mathrm{OPS}_{E}^{m}$, is defined as an operator with a total symbol $a(r, s, t, \xi, \tau)$ admitting an asymptotic expansion in $E$-quasi homogeneous symbols

$$
a(r, s, t, \xi, \tau) \sim \sum_{j=0}^{+\infty} a_{m-j}(r, s, t, \xi, \tau),
$$

where the functions $a_{m-j}, j \in \mathbb{N}$, are $E$-quasi homogeneous of degree $m-j$. The meaning of $\sim$ in (14) is here

$$
\forall \widetilde{m} \in \mathbb{N}, \quad a-\sum_{j=0}^{\widetilde{m}} p_{m-j} \in S_{E}^{m-(\widetilde{m}+1)} .
$$

A symbol $a$ satisfying the property (14) is denoted by $a \in S_{E}^{m}$ and the associated operators $A=O p(a)$ by $A \in \mathrm{OPS}_{E}^{m}$. Finally, we introduce $\mathrm{OPS}_{E}^{-\infty}$ as the intersection between all the classes $\mathrm{OPS}_{E}^{m}, m \in \mathbb{Z}$. For $P$ and $Q$ two pseudodifferential operators with respective symbols $p$ and $q$, and $m \in \mathbb{Z}$, we set

$$
P=Q \quad \bmod \mathrm{OPS}_{E}^{m}
$$

or equivalently

$$
p=q \quad \bmod S_{E}^{m}
$$

if the difference between the two symbols fulfills: $p-q \in S_{E}^{m}$. Finally, the composition formula for two operators $A$ and $B$ with respective symbols $\sigma(A)$ and $\sigma(B)$ writes

$$
\sigma(A B)=\sum_{|\alpha|=0}^{+\infty} \frac{(-i)^{|\alpha|}}{\alpha !} \partial_{(\xi, \tau)}^{\alpha} \sigma(A) \partial_{(s, t)}^{\alpha} \sigma(B) .
$$

Furthermore, if $\sigma(A) \in S_{E}^{m}$ and $\sigma(B) \in S_{E}^{n}$, then we have $\sigma(A B) \in S_{E}^{m+n}$. In (18), $\alpha$ is a multi-index $\left(\alpha_{1}, \alpha_{2}\right)$. We use the classical notations for multi-indices. In particular, its length $|\alpha|$ is defined by: $|\alpha|=\alpha_{1}+\alpha_{2}$. The factorial is defined by: $\alpha !=\alpha_{1} ! \alpha_{2}$ !, and we introduce the derivative according to $(\xi, \tau): \partial_{(\xi, \tau)}^{\alpha} \lambda=\partial_{\xi}^{\alpha_{1}} \partial_{\tau}^{\alpha_{2}} \lambda(r, s, t, \xi, \tau)$. These classes of operators allows to define an associated symbolic calculus $[18,11]$. Finally, we have: $\sigma\left(\partial_{s}\right)=i \xi$ and $\sigma\left(\partial_{s}^{2}\right)=-\xi^{2}$.

\subsection{E-quasi hyperbolic, elliptic and glancing zones}

Let us recall that the ABCs will be built in the high frequency regime. More precisely, we consider the following definition with $E=(1,2)[7]$. 
Definition 2. We define the E-quasi hyperbolic zone as the set $\mathcal{H}$ of points $(s, t, \xi, \tau)$ such that

$$
\mathcal{H}=\left\{(s, t, \xi, \tau),-\tau-\xi^{2}>0\right\}
$$

The construction of the ABCs is then realized under the microlocal assumption that the points $(s, t, \xi, \tau)$ lie in $\mathcal{H}$. This hypothesis characterizes the propagative part of the wave. Two other regions can be also defined: the $E$-quasi elliptic zone $\mathcal{E}$ given by

$$
\mathcal{E}=\left\{(s, t, \xi, \tau),-\tau-\xi^{2}<0\right\},
$$

which gives the evanescent (exponentially decaying) part of the wave and the $E$-quasi glancing zone which is the complementary set $\mathcal{G}$ of $\mathcal{E} \cup \mathcal{H}$. This last region is reduced to $\{0\}$ if $u$ is not tangentially incident to $\Sigma$. We always assume here that the frequencies are defined in $\mathcal{H}$. This assumption is not always valid but is true if we suppose that the evanescent part is reduced to $\{0\}$.

\section{Two possible strategies}

To build the asymptotic expansion of the DtN operator on $\Sigma$, we factorize the Schrödinger operator with Nirenberg's techniques [24] in the generalized system $(r, s)$ given by (8). This allows us, as in the one-dimensional case, to obtain the DtN pseudodifferential operators $\Lambda^{-}$and $\Lambda^{+}$characterizing respectively the incoming and outgoing wave. Two strategies can then be followed according to the fact that the gauge change is applied or not. In each case, we are going to explicit the underlying Schrödinger operator on which a factorization is made.

\subsection{Strategy I: gauge change method}

The first strategy that we consider consists in applying the gauge change before building the boundary condition. Let us recall that the change of unknown (4) leads to the TBC for a potential $V=V(t)$ and a half-space. Let us introduce the phase function $\mathcal{V}$

$$
\mathcal{V}(r, s, t)=\int_{0}^{t} V_{r}(r, s, \sigma) d \sigma
$$

and do the change of unknown

$$
v(r, s, t)=e^{-i \mathcal{V}(r, s, t)} \tilde{u}(r, s, t) .
$$

Then, the new function $v$ is solution to the variable coefficients Schrödinger equation

$$
i \partial_{t} v+\partial_{r}^{2} v+\left(\kappa_{r}+2 i\left(\partial_{r} \mathcal{V}\right)\right) \partial_{r} v+h^{-1} \partial_{s}\left(h^{-1} \partial_{s}\right)+2 i\left(\partial_{s} \mathcal{V}\right) \partial_{s} v+G v=0,
$$

where $G$ is the zero order operator given by

$$
G=i \kappa_{r}\left(\partial_{r} \mathcal{V}\right)+i \partial_{r}^{2} \mathcal{V}-\left(\partial_{r} \mathcal{V}\right)^{2}+i \partial_{s}^{2} \mathcal{V}-\left(\partial_{s} \mathcal{V}\right)^{2}+i h^{-1}\left(\partial_{s} h^{-1}\right)\left(\partial_{s} \mathcal{V}\right)
$$

Hence, after the gauge change, function $v$ is solution to

$$
L\left(r, s, t, \partial_{s}, \partial_{t}\right) v=0, \quad \text { on } \Omega_{T},
$$

with $L$ defined by

$$
L=i \partial_{t}+\partial_{r}^{2}+\left(\kappa_{r}+2 i\left(\partial_{r} \mathcal{V}\right)\right) \partial_{r}+h^{-1} \partial_{s}\left(h^{-1} \partial_{s}\right)+2 i\left(\partial_{s} \mathcal{V}\right) \partial_{s}+G
$$




\subsection{Strategy II: direct method}

The second strategy consists in directly working on the operator involved in (8), that is

$$
L=i \partial_{t}+\partial_{r}^{2}+\kappa_{r} \partial_{r}+h^{-1} \partial_{s}\left(h^{-1} \partial_{s}\right)+V_{r}
$$

Unlike the gauge change method, a term related to the potential will be handled in the principal symbol $\Lambda^{+}$.

Remark 1. As in the one-dimensional case, the difference between the two strategies is related to two points. First, the gauge change (22) is only applied in the first strategy while we directly work on $u$ in the second method. However, a second difference concerns the choice of the principal symbol. In the direct method, and unlike the gauge change, zero order terms are included in the principal symbol to compensate the gauge change effect. Following this strategy, another strategy, called strategy 0 , which is more basic may be considered. This aproach would consist in neither applying the gauge change nor taking the potential into the principal symbol. However, like the one-dimensional case and as seen later (see Remark 4, page 23), this strategy corresponds to a high frequency approximation, for large $|\tau|$, of strategy II via a Taylor expansion. We note that this is however the approach used in $[4,7]$.

\subsection{Unification of the strategies}

In a unified framework, we are now led to build a boundary condition for the Schrödinger equation with variable coefficients $A$ and $B$

$$
\left(i \partial_{t}+\partial_{r}^{2}+\left(\kappa_{r}+A\right) \partial_{r}+h^{-1} \partial_{s}\left(h^{-1} \partial_{s}\right)+B\right) w=0, \quad \text { on } \Omega_{T},
$$

where $A$ and $B$ and the unknown $w$ differ according to strategy I or II. For the gauge change, the unknown is $w=v=e^{-i \mathcal{V}} u$ and

$$
A=2 i\left(\partial_{r} \mathcal{V}\right), \quad B=2 i\left(\partial_{s} \mathcal{V}\right) \partial_{s}+G .
$$

For the direct method, the unknown is $w=u$ and the coefficients are

$$
A=0, \quad B=V_{r} .
$$

\subsection{Symbolic system}

The operator $L$ associated with the general equation (27) writes down

$$
L=i \partial_{t}+\partial_{r}^{2}+\left(\kappa_{r}+A\right) \partial_{r}+h^{-1} \partial_{s}\left(h^{-1} \partial_{s}\right)+B
$$

where the operators $A$ and $B$ are given by either (28) or (29). We have the following factorization theorem.

Theorem 1. Let $L$ be the Schrödinger operator with coefficients (30). There exist two first-order homogeneous classical pseudodifferential operators $\Lambda^{ \pm}=\Lambda^{ \pm}\left(r, s, t, \partial_{s}, \partial_{t}\right) \in O P S_{E}^{1}$, smoothly dependent with respect to $r$, and such that

$$
L\left(r, s, t, \partial_{s}, \partial_{t}\right)=\left(\partial_{r}+i \Lambda^{-}\right)\left(\partial_{r}+i \Lambda^{+}\right)+R
$$


where $R=R\left(r, s, t, \partial_{s}, \partial_{t}\right)$ is a smoothing operator in $O P S_{E}^{-\infty}$. Furthermore, the total symbol $\lambda^{ \pm}=$ $\sigma\left(\Lambda^{ \pm}\right)$of $\Lambda^{ \pm}$admits an asymptotic expansion in E-quasi homogeneous symbols

$$
\sigma\left(\Lambda^{ \pm}\right)=\lambda^{ \pm} \sim \sum_{j=0}^{+\infty} \lambda_{1-j}^{ \pm}
$$

where $\lambda_{1-j}^{ \pm} \in S_{E}^{1-j}$ for $j \in \mathbb{N}$. These asymptotic expansions are unique once the principal symbols $\lambda_{1}^{ \pm}$ are fixed.

Proof. To prove the result, we develop the expression (31) of $L$ and group together the terms in decaying power of $\partial_{r}$

$$
L=\partial_{r}^{2}+i \Lambda^{-} \partial_{r}+i \partial_{r} \Lambda^{+}-\Lambda^{-} \Lambda^{+}+R .
$$

By using the identity $\partial_{r} \Lambda^{+}=\Lambda^{+} \partial_{r}+O p\left(\partial_{r} \lambda^{+}\right)$, we deduce

$$
L=\partial_{r}^{2}+i\left(\Lambda^{+}+\Lambda^{-}\right) \partial_{r}+i O p\left(\partial_{r} \lambda^{+}\right)-\Lambda^{-} \Lambda^{+}+R .
$$

Then, we can compare (30) and (33) and identify the terms of the same order in $\partial_{r}$ in the two expressions, modulo the smoothing operator $R$ of order $-\infty$. We obtain the system

$$
\begin{aligned}
& i\left(\Lambda^{+}+\Lambda^{-}\right)=\kappa_{r}+A \\
& i O p\left(\partial_{r} \lambda^{+}\right)-\Lambda^{-} \Lambda^{+}=i \partial_{t}+h^{-1} \partial_{s}\left(h^{-1} \partial_{s}\right)+B .
\end{aligned}
$$

The term $h^{-1} \partial_{s}\left(h^{-1} \partial_{s}\right)$ can be developed as

$$
h^{-1} \partial_{s}\left(h^{-1} \partial_{s}\right)=h^{-1} h^{-1} \partial_{s}^{2}+h^{-1} \partial_{s}\left(h^{-1}\right) \partial_{s}
$$

implying that the symbol of this operator is

$$
\sigma\left(h^{-1} \partial_{s}\left(h^{-1} \partial_{s}\right)\right)=-h^{-2} \xi^{2}+i h^{-1} \partial_{s}\left(h^{-1}\right) \xi .
$$

Furthermore, the symbol of $\Lambda^{-} \Lambda^{+}$being given by (18), (34) leads to the symbolic system

$$
\begin{aligned}
& i\left(\lambda^{+}+\lambda^{-}\right)=\kappa_{r}+a \\
& i \partial_{r} \lambda^{+}-\sum_{|\alpha|=0}^{+\infty} \frac{(-i)^{|\alpha|}}{\alpha !} \partial_{(\xi, \tau)}^{\alpha} \lambda^{-} \partial_{(s, t)}^{\alpha} \lambda^{+}=-\tau-h^{-2} \xi^{2}+i h^{-1}\left(\partial_{s} h^{-1}\right) \xi+b
\end{aligned}
$$

where $a=A$ and $b=B$, respectively, designate the symbols of the operators $A$ and $B$, respectively. In the gauge change case, we have

$$
a=2 i\left(\partial_{r} \mathcal{V}\right), \quad b=-2\left(\partial_{s} \mathcal{V}\right) \xi+g,
$$

where $g=G$ is the zero order operator defined by (24); for the direct method, the symbols are given by

$$
a=0, \quad b=V_{r} .
$$

We now have to determine the asymptotic expansion in $E$-quasi homogeneous symbols of the total symbol $\lambda^{+}$. This asymptotic expansion is obtained by identifying in (36) the terms of the same order 
of homogeneity. Before that, system (36) can be reduced to one unknown, $\lambda^{+}$. Indeed, the term $a$ only depends on $r, s$ and $t$, but not of $\xi$, nor $\tau$. We first extract $\lambda^{-}$from the first equation

$$
\lambda^{-}=-\lambda^{+}-i \kappa_{r}-i a .
$$

This expression is then injected into the second equation. But, as the terms $\kappa_{r}$ and $a$ do not depend neither on $\tau$ nor on $\xi$, we have

$$
\partial_{(\xi, \tau)}^{\alpha} \lambda^{-}= \begin{cases}-\lambda^{+}-i \kappa_{r}-i a & \text { if } \alpha=0 \\ -\partial_{(\xi, \tau)}^{\alpha} \lambda^{+} & \text {if }|\alpha|>1\end{cases}
$$

In the infinite sum of the second equation of (36), the terms can be written as

$$
\partial_{(\xi, \tau)}^{\alpha} \lambda^{-} \partial_{(s, t)}^{\alpha} \lambda^{+}= \begin{cases}-\lambda^{+} \lambda^{+}-i \kappa_{r} \lambda^{+}-i a \lambda^{+} & \text {if } \alpha=0 \\ -\partial_{(\xi, \tau)}^{\alpha} \lambda^{+} \partial_{(s, t)}^{\alpha} \lambda^{+} & \text {if }|\alpha|>1 .\end{cases}
$$

Hence, the second equation of (36) gives

$$
i \partial_{r} \lambda^{+}+i \kappa_{r} \lambda^{+}+i a \lambda^{+}+\sum_{|\alpha|=0}^{+\infty} \frac{(-i)^{|\alpha|}}{\alpha !} \partial_{(\xi, \tau)}^{\alpha} \lambda^{+} \partial_{(t, r)}^{\alpha} \lambda^{+}=-\tau-h^{-2} \xi^{2}+i h^{-1}\left(\partial_{s} h^{-1}\right) \xi+b .
$$

This last equation will be the starting point to get the symbols $\lambda_{1-j}^{+}, j \in \mathbb{Z}$, which in each case lead to an approximate boundary condition.

The factorization (31) indicates that the reflected part of the wave is given by $w^{+}=\left(\partial_{\mathbf{n}}+i \Lambda^{+}\right) w$. The TBC is then

$$
w^{+}=\left(\partial_{\mathbf{n}}+i \widetilde{\Lambda^{+}}\right) w=0, \quad \text { on } \Sigma_{T},
$$

where the pseudodifferential operator $\widetilde{\Lambda^{+}}$is defined by

$$
\widetilde{\Lambda^{+}}=\Lambda_{\mid r=0}^{+} .
$$

Like $\Lambda^{+}$, the operator $\widetilde{\Lambda^{+}}$can be developed in homogeneous symbols and its expansion is given by

$$
\sigma\left(\widetilde{\Lambda^{+}}\right) \sim \sum_{j=0}^{+\infty} \widetilde{\lambda}_{1-j}
$$

where

$$
\widetilde{\lambda}_{1-j}=\left(\lambda_{1-j}^{+}\right)_{\mid r=0} .
$$

As a consequence, determining the operator $\widetilde{\Lambda^{+}}$requires the computation of its boundary symbols: $\left(\lambda_{1-j}^{+}\right)_{\mid r=0}$. We approximate $\widetilde{\Lambda^{+}}$by truncating its asymptotic expansion (42). Keeping its first $M$ terms $\left(\widetilde{\lambda}_{1-j}\right)_{0 \leq j \leq M-1}$, the approximate boundary condition of order $M$ writes down

$$
\partial_{\mathbf{n}} w_{M}+i \sum_{j=0}^{M-1} O p\left(\widetilde{\lambda}_{1-j}\right) w_{M}=0, \quad \text { on } \Sigma_{T}
$$


where $w_{M}$ is then an approximate solution to (27). In the gauge change case, we then apply the inverse change of unknown $w=e^{-i \mathcal{V}} u$ to express the approximate boundary condition on $u_{M}$. The question is now how to compute the first $M$ symbols of the asymptotic expansion of $\lambda^{+}$, for each strategy and finally evaluating the symbols on the boundary for $r=0$.

\subsection{Adding terms in the principal symbol}

We can classify the terms appearing in the right hand side of (39) in three classes: the second order terms, which must be included in the principal symbol; the potential type terms of order zero which will be considered in the principal symbol only for strategy II; and some first order terms that may be included or not into the principal symbol for both strategies. The key argument allowing to determine if a term may be included in the principal symbol is related to the sign of the imaginary part of $\lambda_{1}^{+}$ which guarantees that the wave is outgoing. As in the one-dimensional case, we have the following lemma.

Lemma 2. The principal symbol $\lambda_{1}^{+}(s, t, \xi, \tau)$ of $\Lambda^{+}$must satisfy the following high frequency condition

$$
\operatorname{Im}\left(\lambda_{1}^{+}(s, t, \xi, \tau)\right) \leq 0, \quad \text { for }|\tau| \gg 1 .
$$

Proof. The idea of the proof is the same as in the one-dimensional case. Let us first consider the case of a principal symbol of the form: $\lambda_{1}^{+}=\lambda_{1}^{+}(\xi, \tau)$, corresponding to the tangent plane approximation. The artificial boundary condition at $(x, y)$ of $\Sigma$, described by its local coordinates $(r, s)$, is given by

$$
\partial_{r} w(r, s, t)+i O p\left(\lambda^{+}\right) w(r, s, t)=0, \quad \text { for } t \in[0 ; T] .
$$

The first symbol being the leading order term in the asymptotics of $\lambda^{+}$, we have the following first order approximation

$$
\partial_{r} w(r, s, t)+i O p\left(\lambda_{1}^{+}\right) w(r, s, t)=0, \quad \text { for } t \in[0 ; T] .
$$

Applying the partial Fourier transform according to $(s, t)$ to this equation leads by definition of the symbol of an operator to

$$
\partial_{r} \hat{w}(r, \xi, \tau)+i \lambda_{1}^{+}(\xi, \tau) \hat{w}(r, \xi, \tau)=0, \quad(\xi, \tau) \in \mathbb{R}^{2} .
$$

Integrating this ordinary differential equation with respect to $r$ gives

$$
\hat{w}(r, \xi, \tau)=A e^{-i \lambda_{1}^{+}(\xi, \tau) r} \quad \text { for }(\xi, \tau) \in \mathbb{R}^{2} .
$$

A $L^{2}(\Omega)$ integrability condition for the frequencies in the elliptic zone $\mathcal{E}$ defined by (20) gives that $\operatorname{Re}\left(-i \lambda_{1}^{+}\right) \leq 0$. Since $\operatorname{Re}\left(-i \lambda_{1}^{+}\right)=\operatorname{Im}\left(\lambda_{1}^{+}\right)$, we deduce condition (44). If $\lambda_{1}^{+}=\lambda_{1}^{+}(r, s, t, \xi, \tau)$ also depends on $r, s$ and $t$, we consider large $|\tau|$ and we asymptotically get

$$
\lambda_{1}^{+}(r, s, t, \xi, \tau) \sim_{|\tau| \rightarrow \infty} \lambda_{1}^{+}\left(r_{0}, s_{0}, t_{0}, \xi, \tau\right),
$$

where the points $r_{0}, s_{0}$ and $t_{0}$ are given. Similarly, we obtain on $\lambda_{1}^{+}\left(x_{0}, t_{0}, \tau\right)$ the necessary condition $\operatorname{Im}\left(\lambda_{1}^{+}\left(r_{0}, s_{0}, t_{0}, \xi, \tau\right)\right) \leq 0$ for large $|\tau|$. We deduce (44). 
As a conclusion, we may include in $\lambda_{1}^{+}$

- real valued terms (as for example the potential) without sign restriction;

- complex valued terms as soon as their imaginary part has a fixed sign: a complex valued term with a positive imaginary sign will lead to choose the negative sign in front of the square-root defining $\lambda_{1}^{+}$while a negative imaginary part will lead to a plus sign. If the imaginary part of the complex term has no sign, then we cannot characterize the outgoing wave. This case will not be considered in the sequel.

\section{$5 \quad$ Strategy I: gauge change}

In this strategy, we made the change of unknown (22) and we must solve the equation (39)

$$
\begin{aligned}
i \partial_{r} \lambda^{+}+i \kappa_{r} \lambda^{+}-2\left(\partial_{r} \mathcal{V}\right) \lambda^{+}+\sum_{|\alpha|=0}^{+\infty} \frac{(-i)^{|\alpha|}}{\alpha !} \partial_{(\xi, \tau)}^{\alpha} \lambda^{+} \partial_{(t, r)}^{\alpha} \lambda^{+} \\
=-\tau-h^{-2} \xi^{2}+i h^{-1}\left(\partial_{s} h^{-1}\right) \xi-2\left(\partial_{s} \mathcal{V}\right) \xi+g
\end{aligned}
$$

with

$$
g=i \kappa_{r} \partial_{r} \mathcal{V}+i \partial_{r}^{2} \mathcal{V}-\left(\partial_{r} \mathcal{V}\right)^{2}+i \partial_{s}^{2} \mathcal{V}-\left(\partial_{s} \mathcal{V}\right)^{2}+i h^{-1} \partial_{s} h^{-1} \partial_{s} \mathcal{V}
$$

\subsection{Choice of the principal symbol}

The first question concerns the choice of the principal symbol which directly impacts the symbolic calculus. We obtain the principal symbol by identifying the second order terms in the equation (46). In the left hand side, the second order term is: $\left(\lambda_{1}^{+}\right)^{2}$. However, different solution exist for the right hand side. Strictly, the second order term is: $-\tau-h^{-2} \xi^{2}$, leading to the principal symbol

$$
\lambda_{1}^{+}= \pm \sqrt{-\tau-h^{-2} \xi^{2}} .
$$

The characterization of the outgoing wave leads to choose the correct sign. However, an inhomogeneous point of view allows to handle in the principal symbol $\lambda_{1}^{+}$any lower order term, like for example in the one-dimensional case where we fixed the principal symbol to $\lambda_{1 / 2}^{+}=-\sqrt{-\tau+V}$. Initially, many possibilities can be adopted for the principal symbol if we include or not the terms $i h^{-1}\left(\partial_{s} h^{-1}\right) \xi$ and/or $-2\left(\partial_{s} \mathcal{V}\right) \xi$ and/or $g$. To guarantee that $\lambda_{1}^{+}$corresponds to an outgoing wave characterization, we must check that condition (44) holds. This implies that complex valued terms with unsigned imaginary part cannot be considered in the square-root. This is typically the case of the first and third terms. The term $-2 \partial_{s} \mathcal{V} \xi$ can be included since it is real valued. Finally, two admissible solution may be considered

$$
\lambda_{1}^{+}=-\sqrt{-\tau-h^{-2} \xi^{2}}
$$

and

$$
\lambda_{1}^{+}=-\sqrt{-\tau-h^{-2} \xi^{2}-2 \partial_{s} \mathcal{V} \xi}
$$


The main difference between symbol (48) and all the other symbols is that its evaluation at $r=0$ always include a term involving $\xi$

$$
\tilde{\lambda}_{1}=-\sqrt{-\tau-\xi^{2}-2\left(\partial_{s} \mathcal{V}\right) \xi} .
$$

This term is multiplied by the tangential variation of the phase function $\mathcal{V}$ which has an a priori relatively small amplitude since the radial variations of the potential are generally larger. The extreme case is the radial potential $V(r, t)$ since $\partial_{s} \mathcal{V}=0$. Hence, the information contained in the term $2\left(\partial_{s} \mathcal{V}\right) \xi$ can be considered as relatively limited and will not be included inside the principal symbol. It will however appear in $\lambda_{0}^{+}$when identifying the first order terms in the equation. This precisely corresponds to the choice (47) as principal symbol.

Concerning the gauge change, only the asymptotics given by (47) will be considered. Two approaches are then derived for each strategy, one based on a Taylor expansion for high frequencies $|\tau|$, and the other one based on square roots of operators which will then be numerically approximated by Padé functions. Even if the Padé approximation itself is practically treated in [6], we will always refer to as Padé approach for strategy II.

\subsection{Symbols computation}

The principal symbol $\lambda_{1}^{+}$being fixed by (47), we successively compute the next symbols following the symbolic calculus rules for the two-dimensional situation. To obtain $\lambda_{0}^{+}$, we consider the first order terms in $(46)$

$$
i \partial_{r} \lambda_{1}^{+}+i \kappa_{r} \lambda_{1}^{+}-2\left(\partial_{r} \mathcal{V}\right) \lambda_{1}^{+}+2 \lambda_{1}^{+} \lambda_{0}^{+}-i \partial_{\xi} \lambda_{1}^{+} \partial_{s} \lambda_{1}^{+}=i h^{-1} \partial_{s} h^{-1} \xi
$$

giving $\lambda_{0}^{+}$

$$
\lambda_{0}^{+}=-\frac{i}{2} \kappa_{r}+\partial_{r} \mathcal{V}+\frac{1}{2 \lambda_{1}^{+}}\left(-i \partial_{r} \lambda_{1}^{+}+i \partial_{\xi} \lambda_{1}^{+} \partial_{s} \lambda_{1}^{+}+i h^{-1} \partial_{s} h^{-1} \xi-2 \partial_{s} \mathcal{V} \xi\right)
$$

and then

$$
\lambda_{0}^{+}=-\frac{i}{2} \kappa_{r}+\partial_{r} \mathcal{V}-\frac{i}{2} \frac{h^{-1}\left(\partial_{s} h^{-1}\right) \xi}{\sqrt{-\tau-h^{-2} \xi^{2}}}+\frac{\left(\partial_{s} \mathcal{V}\right) \xi}{\sqrt{-\tau-h^{-2} \xi^{2}}}-\frac{i}{2} \frac{h^{-3}\left(\partial_{r} h\right) \xi^{2}}{-\tau-h^{-2} \xi^{2}}+\frac{i}{2} \frac{h^{-5}\left(\partial_{s} h\right) \xi^{3}}{\sqrt{-\tau-h^{-2} \xi^{2}}}
$$

Next, we compute $\lambda_{-1}^{+}$and $\lambda_{-2}^{+}$. Since the calculations are too cumbersome, we do not give the expressions of these symbols. We only report $\widetilde{\lambda}_{j}$, which are the evaluations of $\lambda_{j}^{+}$at $r=0$

$$
\begin{aligned}
\tilde{\lambda}_{1}= & -\sqrt{-\tau-\xi^{2}}, \\
\tilde{\lambda}_{0}= & -\frac{i}{2} \kappa+\partial_{\mathbf{n}} \mathcal{V}+\frac{\left(\partial_{s} \mathcal{V}\right) \xi}{\sqrt{-\tau-\xi^{2}}}-\frac{i}{2} \frac{\kappa \xi^{2}}{-\tau-\xi^{2}} \\
\tilde{\lambda}_{-1}= & -\frac{1}{8} \frac{\kappa^{2}}{\sqrt{-\tau-\xi^{2}}}-\frac{1}{2} \frac{\left(\partial_{s} \kappa\right) \xi}{\sqrt{-\tau-\xi^{2}}}-\frac{1}{2} \frac{i \partial_{s}^{2} \mathcal{V}-\left(\partial_{s} \mathcal{V}\right)^{2}}{\sqrt{-\tau-\xi^{2}}}-\frac{1}{2} \frac{\left(i \partial_{s}^{2} \mathcal{V}-\left(\partial_{s} \mathcal{V}\right)^{2}\right) \xi^{2}}{{\sqrt{-\tau-\xi^{2}}}^{3}} \\
& -\frac{3}{4} \frac{\xi^{2} \kappa^{2}}{\sqrt{-\tau-\xi^{2}}}-i \frac{\kappa\left(\partial_{s} \mathcal{V}\right) \xi^{3}}{\left(-\tau-\xi^{2}\right)^{2}}-\frac{1}{2} \frac{\left(\partial_{s} \kappa\right) \xi^{3}}{\left(-\tau-\xi^{2}\right)^{2}}-\frac{5}{8} \frac{\kappa^{2} \xi^{4}}{{\sqrt{-\tau-\xi^{2}}}^{5}} \\
\tilde{\lambda}_{-2}= & \frac{i}{8} \frac{\kappa^{3}}{-\tau-\xi^{2}}+\frac{i}{8} \frac{\partial_{s}^{2} \kappa}{-\tau-\xi^{2}}-\frac{i}{4} \frac{\partial_{\mathbf{n}} V}{-\tau-\xi^{2}}-\frac{1}{2} \frac{\left(\partial_{s} \kappa\right)\left(\partial_{s} \mathcal{V}\right)}{-\tau-\xi^{2}}+\ldots
\end{aligned}
$$


Remark 2. Since many terms appear in $\widetilde{\lambda}_{-2}$, we do not consider all of them. All the terms in $\widetilde{\lambda}_{-2}$ are $E$-quasi homogeneous of order -2 but can also be written either as the ratio between a zero order term and a symbol of order 2 , or as the ratio between a first order symbol and a symbol of order 3 and so on. The common point is that the terms in $\tau$ only appear in the denominator. In the Taylor expansion of the symbols, the asymptotics will be made according to large values of $|\tau|$ in the $E$-quasi hyperbolic zone $\mathcal{H}$. For the fourth order condition (the highest order considered in the present paper), we will only keep terms in at least $\tau^{-1}$ and higher. Since the terms in the expression of $\widetilde{\lambda}_{-2}$ have a denominator of the form ${\sqrt{-\tau-\xi^{2}}}^{3},\left(-\tau-\xi^{2}\right)^{2}, \ldots$, leading to terms of the order of $\tau^{-3 / 2}, \tau^{-2}, \ldots$ in the Taylor expansion, they will not be considered in the fourth order condition. Finally, the terms which are not given will not be considered in the writing of the boundary conditions until the fifth order for the Taylor approach. They are not necessary either in the Padé approximation since this approximation is more accurate. For this reason, only artificial boundary conditions taking $\widetilde{\lambda}_{1}$ and $\widetilde{\lambda}_{0}$ will be considered in the Padé approach.

Considering the symbols (50)-(53), we get the boundary conditions of order $M, M \in\{1,2,3,4\}$, for $v$, given by

$$
\partial_{\mathbf{n}} v+i \sum_{j=0}^{M-1} O p\left(\widetilde{\lambda}_{1-j}\right) v=0, \quad \text { on } \Sigma_{T} .
$$

In terms of unknown $u$, we have the $\mathrm{ABC}$ of order $M$

$$
\partial_{\mathbf{n}} u-i \partial_{\mathbf{n}} \mathcal{V} u+i e^{i \mathcal{V}} \sum_{j=0}^{M-1} O p\left(\widetilde{\lambda}_{1-j}\right)\left(e^{-i \mathcal{V}} u\right)=0, \quad \text { on } \Sigma_{T},
$$

where $\mathcal{V}$ is the phase function. The next step consists in interpreting the operators $O p\left(\tilde{\lambda}_{j}\right)$ in such a way that (54) is written in terms of explicit operators. This is done for both the Taylor and Padé approaches.

\subsection{Interpretation of the ABCs for the Taylor approach}

In this approach which is also considered in $[4,7]$, we make a high frequency asymptotic expansion of the symbols for $|\tau| \gg \xi^{2}$ in the $E$-quasi hyperbolic zone $\mathcal{H}$. Let us introduce

$$
\tilde{\lambda}_{1-j}=\left(\tilde{\lambda}_{1-j}\right)_{(\alpha)}+O\left(\tau^{\alpha-1 / 2}\right),
$$

where $\left(\tilde{\lambda}_{1-j}\right)_{(\alpha)}$ designates the truncation of the Taylor expansion of $\tilde{\lambda}_{1-j}$ by limiting to terms of order at least equal to $\alpha$. From a general point of view, the order $M$ condition is given by

$$
\partial_{\mathbf{n}} u-i \partial_{\mathbf{n}} \mathcal{V} u+i e^{i \mathcal{V}} \sum_{j=0}^{+\infty} O p\left(\left(\widetilde{\lambda}_{1-j}\right)_{(1-M / 2)}\right)\left(e^{-i \mathcal{V}} u\right)=0, \quad \text { on } \Sigma_{T}
$$

However, this sum is in fact finite since the terms of order $1-M / 2$ are only contained in $\widetilde{\lambda}_{j}$, for $1 \leq j \leq 2-M$. The order $M$ boundary condition writes down

$$
\partial_{\mathbf{n}} u-i \partial_{\mathbf{n}} \mathcal{V} u+i e^{i \mathcal{V}} \sum_{j=0}^{M-1} O p\left(\left(\widetilde{\lambda}_{1-j}\right)_{(1-M / 2)}\right)\left(e^{-i \mathcal{V}} u\right)=0, \quad \text { on } \Sigma_{T}
$$


In the sequel, we denote this $\mathrm{ABC}$ by $\mathrm{ABC}_{1, T}^{M}$, the index 1 refereeing to as the first strategy and the index $T$ to the Taylor approach. By using the symbols $\widetilde{\lambda}_{1}, \widetilde{\lambda}_{0}, \widetilde{\lambda}_{-1}$ and $\widetilde{\lambda}_{-2}$, we can write four boundary conditions, from the first order condition

$$
\partial_{\mathbf{n}} u-i \partial_{\mathbf{n}} \mathcal{V} u+i e^{i \mathcal{V}} O p\left(\left(\widetilde{\lambda}_{1}\right)_{(1 / 2)}\right)\left(e^{-i \mathcal{V}} u\right)=0, \quad \text { on } \Sigma_{T}
$$

to the fourth order one

$$
\partial_{\mathbf{n}} u-i \partial_{\mathbf{n}} \mathcal{V} u+i e^{i \mathcal{V}} O p\left(\left(\widetilde{\lambda}_{1}\right)_{(-1)}+\left(\widetilde{\lambda}_{0}\right)_{(-1)}+\left(\widetilde{\lambda}_{-1}\right)_{(-1)}+\left(\widetilde{\lambda}_{-2}\right)_{(-1)}\right)\left(e^{-i \mathcal{V}} u\right)=0, \quad \text { on } \Sigma_{T}
$$

Since we have

$$
\begin{aligned}
& \tilde{\lambda}_{1}=-\sqrt{-\tau}+\frac{\xi^{2}}{2 \sqrt{-\tau}}+O\left(\frac{1}{\tau^{3 / 2}}\right) \\
& \tilde{\lambda}_{0}=-\frac{i}{2} \kappa+\partial_{\mathbf{n}} \mathcal{V}+\frac{\partial_{s} \mathcal{V} \xi}{\sqrt{-\tau}}+\frac{i}{2} \frac{\kappa \xi^{2}}{\tau}+O\left(\frac{1}{\tau^{2}}\right) \\
& \tilde{\lambda}_{-1}=-\frac{\kappa^{2}}{8} \frac{1}{\sqrt{-\tau}}-\frac{1}{2} \frac{i \partial_{s}^{2} \mathcal{V}-\left(\partial_{s} \mathcal{V}\right)^{2}}{\sqrt{-\tau}}+\frac{1}{2} \frac{\partial_{s} \kappa \xi}{\tau}+O\left(\frac{1}{\tau^{3 / 2}}\right), \\
& \tilde{\lambda}_{-2}=\frac{i}{4} \frac{\partial_{\mathbf{n}} V}{\tau}-\frac{i}{8} \frac{\partial_{s}^{2} \kappa}{\tau}-\frac{i}{8} \frac{\kappa^{3}}{\tau}+\frac{1}{2} \frac{\partial_{s} \kappa \partial_{s} \mathcal{V}}{\tau}+O\left(\frac{1}{\tau^{3 / 2}}\right),
\end{aligned}
$$

the truncated symbols are given by

$$
\begin{aligned}
& \left(\tilde{\lambda}_{1}\right)_{(-1)}=-\sqrt{-\tau}+\frac{\xi^{2}}{2 \sqrt{-\tau}}, \\
& \left(\tilde{\lambda}_{0}\right)_{(-1)}=-\frac{i}{2} \kappa+\partial_{\mathbf{n}} \mathcal{V}+\frac{\partial_{s} \mathcal{V} \xi}{\sqrt{-\tau}}+\frac{i}{2} \frac{\kappa \xi^{2}}{\tau} \\
& \left(\tilde{\lambda}_{-1}\right)_{(-1)}=-\frac{\kappa^{2}}{8} \frac{1}{\sqrt{-\tau}}-\frac{1}{2} \frac{i \partial_{s}^{2} \mathcal{V}-\left(\partial_{s} \mathcal{V}\right)^{2}}{\sqrt{-\tau}}+\frac{1}{2} \frac{\partial_{s} \kappa \xi}{\tau} \\
& \left(\tilde{\lambda}_{-2}\right)_{(-1)}=\frac{i}{4} \frac{\partial_{\mathbf{n}} V}{\tau}-\frac{i}{8} \frac{\partial_{s}^{2} \kappa}{\tau}-\frac{i}{8} \frac{\kappa^{3}}{\tau}+\frac{1}{2} \frac{\partial_{s} \kappa \partial_{s} \mathcal{V}}{\tau}
\end{aligned}
$$

Let us now write the fourth order condition (the lowest order ones can be deduced directly) for the function $v$

$$
\begin{aligned}
\partial_{\mathbf{n}} v-i O p(\sqrt{-\tau}) v+\frac{\kappa}{2} v+ & i \partial_{\mathbf{n}} \mathcal{V} v-i O p\left(\left(\frac{\kappa^{2}}{8}-\frac{\xi^{2}}{2}-\partial_{s} \mathcal{V} \xi+\frac{1}{2}\left(i \partial_{s}^{2} \mathcal{V}-\left(\partial_{s} \mathcal{V}\right)^{2}\right)\right) \frac{1}{\sqrt{-\tau}}\right) v \\
+ & O p\left(\left(-\frac{\kappa \xi^{2}}{2}+\frac{i \partial_{s} \kappa \xi}{2}+\frac{\kappa^{3}}{8}+\frac{\partial_{s}^{2} \kappa}{8}-\frac{\partial_{\mathbf{n}} V}{4}+\frac{i \partial_{s} \kappa \partial_{s} \mathcal{V}}{2}\right) \frac{1}{\tau}\right) v=0
\end{aligned}
$$


Since $v=e^{-i \mathcal{V}} u\left(\right.$ and $\left.e^{i \mathcal{V}} \partial_{\mathbf{n}} v=\partial_{\mathbf{n}} u-i \partial_{\mathbf{n}} \mathcal{V} u\right)$ we obtain

$$
\begin{aligned}
\partial_{\mathbf{n}} u-i e^{i \mathcal{V}} O p(\sqrt{-\tau})\left(e^{-i \mathcal{V}} u\right)+\frac{\kappa}{2} u \\
-i e^{i \mathcal{V} O p}\left(\left(\frac{\kappa^{2}}{8}-\frac{\xi^{2}}{2}-\partial_{s} \mathcal{V} \xi+\frac{1}{2}\left(i \partial_{s}^{2} \mathcal{V}-\left(\partial_{s} \mathcal{V}\right)^{2}\right)\right) \frac{1}{\sqrt{-\tau}}\right)\left(e^{-i \mathcal{V}} u\right) \\
\quad+e^{i \mathcal{V}} O p\left(\left(-\frac{\kappa \xi^{2}}{2}+\frac{i \partial_{s} \kappa \xi}{2}+\frac{\kappa^{3}}{8}+\frac{\partial_{s}^{2} \kappa}{8}-\frac{\partial_{\mathbf{n}} V}{4}+\frac{i \partial_{s} \kappa \partial_{s} \mathcal{V}}{2}\right) \frac{1}{\tau}\right)\left(e^{-i \mathcal{V}} u\right)=0
\end{aligned}
$$

It remains now to interpret the operators given by their symbols. Hereabove, the surface laplacien $\Delta_{\Sigma}$ is a constant coefficients surface operator on $\Sigma$ since $\Delta_{\Sigma}=\left(\Delta_{r}\right)_{\mid r=0}$ (the surface laplacian corresponds to the evaluation on the boundary of the laplacian written in local coordinates). Since we are working in a two-dimensional setting and that we can use a global parameterization of the boundary, only $\Delta_{\Sigma}=\partial_{s}^{2}$ remains. This would not hold for the three-dimensional case. We finally have

$$
O p\left(-\xi^{2}\right)=\Delta_{\Sigma}, \quad O p(-\xi)=i \partial_{s}, \quad O p\left(-\kappa \xi^{2}+i \partial_{s} \kappa \xi\right)=\kappa \partial_{s}^{2}+\partial_{s} \kappa \partial_{s}=\partial_{s}\left(\kappa \partial_{s}\right) .
$$

Furthermore, symbols in $\tau$ lead to

$$
O p(-i \sqrt{-\tau})=e^{-i \pi / 4} \partial_{t}^{1 / 2}, \quad O p\left(\frac{-i}{\sqrt{-\tau}}\right)=-e^{i \pi / 4} I_{t}^{1 / 2}, \quad O p\left(\frac{1}{\tau}\right)=i I_{t} .
$$

The fourth order $\mathrm{ABC}$ is then

$$
\begin{aligned}
\partial_{\mathbf{n}} u+e^{-i \pi / 4} e^{i \mathcal{V}} \partial_{t}^{1 / 2}\left(e^{-i \mathcal{V}} u\right) & +\frac{\kappa}{2} u \\
-e^{i \pi / 4} e^{i \mathcal{V}}\left(\frac{\kappa^{2}}{8}+\right. & \left.\frac{\Delta_{\Sigma}}{2}+i \partial_{s} \mathcal{V} \partial_{s}+\frac{1}{2}\left(i \partial_{s}^{2} \mathcal{V}-\left(\partial_{s} \mathcal{V}\right)^{2}\right)\right) I_{t}^{1 / 2}\left(e^{-i \mathcal{V}} u\right) \\
& +i e^{i \mathcal{V}}\left(\frac{\partial_{s}\left(\kappa \partial_{s}\right)}{2}+\frac{\kappa^{3}+\partial_{s}^{2} \kappa}{8}-\frac{\partial_{\mathbf{n}} V}{4}+\frac{i \partial_{s} \kappa \partial_{s} \mathcal{V}}{2}\right) I_{t}\left(e^{-i \mathcal{V}} u\right)=0
\end{aligned}
$$

In the sequel, we will try to obtain a priori estimates. For this reason and as in the one-dimensional case, a symetrized version of the $\mathrm{ABC}$ is preferred. This leads us to symmetrize the term which depends on the potential $\partial_{\mathbf{n}} V / 4$ by writing $\partial_{\mathbf{n}} V=\operatorname{sg}\left(\partial_{\mathbf{n}} V\right) \sqrt{\left|\partial_{\mathbf{n}} V\right|} \sqrt{\left|\partial_{\mathbf{n}} V\right|}$. The fourth order ABC, denoted by $\mathrm{ABC}_{1, T}^{4}$, is then given by

$$
\begin{aligned}
& \partial_{\mathbf{n}} u+e^{-i \pi / 4} e^{i \mathcal{V}} \partial_{t}^{1 / 2}\left(e^{-i \mathcal{V}} u\right)+\frac{\kappa}{2} u \\
&-e^{i \pi / 4} e^{i \mathcal{V}}\left(\frac{\kappa^{2}}{8}+\frac{\Delta_{\Sigma}}{2}+i \partial_{s} \mathcal{V} \partial_{s}+\frac{1}{2}\left(i \partial_{s}^{2} \mathcal{V}-\left(\partial_{s} \mathcal{V}\right)^{2}\right)\right) I_{t}^{1 / 2}\left(e^{-i \mathcal{V}} u\right) \\
& \quad+i e^{i \mathcal{V}}\left(\frac{\partial_{s}\left(\kappa \partial_{s}\right)}{2}+\frac{\kappa^{3}+\partial_{s}^{2} \kappa}{8}+\frac{i \partial_{s} \kappa \partial_{s} \mathcal{V}}{2}\right) I_{t}\left(e^{-i \mathcal{V}} u\right) \\
& \quad-i \frac{\operatorname{sg}\left(\partial_{\mathbf{n}} V\right)}{4} \sqrt{\left|\partial_{\mathbf{n}} V\right|} e^{i \mathcal{V}} I_{t}\left(\sqrt{\left|\partial_{\mathbf{n}} V\right|} e^{-i \mathcal{V}} u\right)=0 .
\end{aligned}
$$

We can clearly extract lower orders ABCs from this construction. These ABCs will be discretized by using discrete convolutions associated with the operators $\partial_{t}^{1 / 2}, I_{t}^{1 / 2}$ and $I_{t}[5,6,7,4]$. The results are summarized in Proposition 1. 
Proposition 1. In strategy I and following Taylor expansions, the ABCs of order $M$ are given by

$$
\partial_{\mathbf{n}} u+\Lambda_{1, T}^{M} u=0, \quad \text { on } \Sigma_{T},
$$

where the operators $\Lambda_{1, T}^{M}$ are defined on $\Sigma_{T}$ by

$$
\begin{aligned}
& \Lambda_{1, T}^{2} u=e^{-i \pi / 4} e^{i \mathcal{V}} \partial_{t}^{1 / 2}\left(e^{-i \mathcal{V}} u\right)+\frac{\kappa}{2} u \\
& \Lambda_{1, T}^{3} u=\Lambda_{1, T}^{2} u-e^{i \pi / 4} e^{i \mathcal{V}}\left(\frac{\kappa^{2}}{8}+\frac{\Delta_{\Sigma}}{2}+i \partial_{s} \mathcal{V} \partial_{s}+\frac{1}{2}\left(i \partial_{s}^{2} \mathcal{V}-\left(\partial_{s} \mathcal{V}\right)^{2}\right)\right) I_{t}^{1 / 2}\left(e^{-i \mathcal{V}} u\right) \\
& \Lambda_{1, T}^{4} u=\Lambda_{1, T}^{3} u+i e^{i \mathcal{V}}\left(\frac{\partial_{s}\left(\kappa \partial_{s}\right)}{2}+\frac{\kappa^{3}+\partial_{s}^{2} \kappa}{8}+\frac{i \partial_{s} \kappa \partial_{s} \mathcal{V}}{2}\right) I_{t}\left(e^{-i \mathcal{V}} u\right) \\
&-i \frac{\operatorname{sg}\left(\partial_{\mathbf{n}} V\right)}{4} \sqrt{\left|\partial_{\mathbf{n}} V\right|} e^{i \mathcal{V}} I_{t}\left(\sqrt{\left|\partial_{\mathbf{n}} V\right|} e^{-i \mathcal{V}} u\right)
\end{aligned}
$$

and where the phase function $\mathcal{V}$ is given by

$$
\mathcal{V}(x, y, t)=\int_{0}^{t} V(x, y, \sigma) d \sigma .
$$

These $A B C$ s are denoted by $A B C_{1, T}^{M}$ in the sequel.

\subsection{Interpretation of the ABCs for the Padé approach}

We can also write the boundary conditions by keeping the symbols and approximating them by Padé approximants in view of an efficient numerical implementation. We will define the $\mathrm{ABC}$ of order $M$ as the boundary condition simply obtained by retaining the first $M$ symbols

$$
\partial_{\mathbf{n}} u-i \partial_{\mathbf{n}} \mathcal{V}+i e^{i \mathcal{V}} \sum_{j=0}^{M-1} O p\left(\widetilde{\lambda}_{1-j}\right)\left(e^{-i \mathcal{V}} u\right)=0, \quad \text { on } \Sigma_{T}
$$

The boundary condition is denoted by $\mathrm{ABC}_{1, P}^{M}$. Since the exact symbols contain more information than for the Taylor approach, we will (arbitrarily) keep less terms leading to lower order boundary conditions. The second order boundary condition which considers the two first symbols $\tilde{\lambda}_{1}$ and $\tilde{\lambda}_{0}$ writes down

$$
\partial_{\mathbf{n}} v-i O p\left(\sqrt{-\tau-\xi^{2}}\right) v+\frac{\kappa}{2} v-\partial_{\mathbf{n}} \mathcal{V} v+i O p\left(\frac{\partial_{s} \mathcal{V} \xi}{\sqrt{-\tau-\xi^{2}}}\right) v+\frac{1}{2} O p\left(\frac{\kappa \xi^{2}}{-\tau-\xi^{2}}\right) v=0
$$

Going back to $u$ by the change of unknown, we obtain

$$
\begin{aligned}
\partial_{\mathbf{n}} u-i e^{i \mathcal{V}} O p\left(\sqrt{-\tau-\xi^{2}}\right) & \left(e^{-i \mathcal{V}} u\right)+\frac{\kappa}{2} u \\
& +e^{i \mathcal{V}} O p\left(\frac{i \partial_{s} \mathcal{V} \xi}{\sqrt{-\tau-\xi^{2}}}\right)\left(e^{-i \mathcal{V}} u\right)+\frac{1}{2} e^{i \mathcal{V}} O p\left(\frac{\kappa \xi^{2}}{-\tau-\xi^{2}}\right)\left(e^{-i \mathcal{V}} u\right)=0
\end{aligned}
$$


We have the following link between the symbols and the operators

$$
\begin{gathered}
O p\left(\sqrt{-\tau-\xi^{2}}\right)=\sqrt{i \partial_{t}+\Delta_{\Sigma}}, \\
O p\left(\frac{i \xi}{\sqrt{-\tau-\xi^{2}}}\right)=\partial_{s}\left(i \partial_{t}+\Delta_{\Sigma}\right)^{-1 / 2}=\left(i \partial_{t}+\Delta_{\Sigma}\right)^{-1 / 2} \partial_{s}, \\
O p\left(\frac{\xi^{2}}{-\tau-\xi^{2}}\right)=-\Delta_{\Sigma}\left(i \partial_{t}+\Delta_{\Sigma}\right)^{-1}=-\left(i \partial_{t}+\Delta_{\Sigma}\right)^{-1} \Delta_{\Sigma} .
\end{gathered}
$$

Here, since the operators to identify are independent of $s$ and $t$, these substitutions are exact unlike what we will observe in the second strategy. Hence, we have the second order boundary condition

$$
\begin{aligned}
\partial_{\mathbf{n}} u-i e^{i \mathcal{V}} \sqrt{i \partial_{t}+\Delta_{\Sigma}}\left(e^{-i \mathcal{V}} u\right)+\frac{\kappa}{2} u+\partial_{s} \mathcal{V} e^{i \mathcal{V}} \partial_{s}\left(i \partial_{t}+\Delta_{\Sigma}\right)^{-1 / 2}\left(e^{-i \mathcal{V}} u\right) & -\frac{\kappa}{2} e^{i \mathcal{V}}\left(i \partial_{t}+\Delta_{\Sigma}\right)^{-1} \Delta_{\Sigma}\left(e^{-i \mathcal{V}} u\right)=0 .
\end{aligned}
$$

The first order boundary condition is deduced by truncation. We do not give the higher order boundary conditions which would not be useful for computational purpose. The following proposition summarizes the results.

Proposition 2. For strategy I and following a Padé approximation, the ABCs of order $M$ are given by

$$
\partial_{\mathbf{n}} u+\Lambda_{1, P}^{M} u=0, \quad \text { on } \Sigma_{T}
$$

where the operators $\Lambda_{1, P}^{M}$ are defined on $\Sigma_{T}$ by

$$
\begin{aligned}
& \Lambda_{1, P}^{1} u=-i e^{i \mathcal{V}} \sqrt{i \partial_{t}+\Delta_{\Sigma}}\left(e^{-i \mathcal{V}} u\right), \\
& \Lambda_{1, P}^{2} u=\Lambda_{1, P}^{1} u+\frac{\kappa}{2} u+\partial_{s} \mathcal{V} e^{i \mathcal{V}} \partial_{s}\left(i \partial_{t}+\Delta_{\Sigma}\right)^{-1 / 2}\left(e^{-i \mathcal{V}} u\right)-\frac{\kappa}{2} e^{i \mathcal{V}}\left(i \partial_{t}+\Delta_{\Sigma}\right)^{-1} \Delta_{\Sigma}\left(e^{-i \mathcal{V}} u\right)
\end{aligned}
$$

We specify these boundary conditions by $A B C_{1, P}^{M}$.

Remark 3. Looking at (66), two equivalent interpretations are possible for $\lambda_{-2}^{+}$: $\Delta_{\Sigma}\left(i \partial_{t}+\Delta_{\Sigma}\right)^{-1}$ or $\left(i \partial_{t}+\Delta_{\Sigma}\right)^{-1} \Delta_{\Sigma}$. We choose the second solution for implementation issues in a finite element framework [6].

\section{Strategy II: direct method}

In this strategy, we directly work on the equation (8) and we must solve the equation in $\lambda^{+}$given by (39)

$$
i \partial_{r} \lambda^{+}+i \kappa_{r} \lambda^{+}+\sum_{|\alpha|=0}^{+\infty} \frac{(-i)^{|\alpha|}}{\alpha !} \partial_{(\xi, \tau)}^{\alpha} \lambda^{+} \partial_{(t, r)}^{\alpha} \lambda^{+}=-\tau-h^{-2} \xi^{2}+i h^{-1}\left(\partial_{s} h^{-1}\right) \xi+V_{r}
$$




\subsection{Computation of the symbols}

Let us begin by fixing the principal symbol. This can be achieved by extracting the second order terms in (71). Like the gauge change, we must determine which second order terms in the right hand side of (71) must be retained. The direct method consists in including the potential in the principal symbol to compensate the gauge change effect. We exclude the case $\lambda_{1}^{+}= \pm \sqrt{-\tau-h^{-2} \xi^{2}}$ (which corresponds to the zero order strategy in Remark 1 leading to less accurate ABCs). Considering the term $i h^{-1} \partial_{s} h^{-1} \xi$ is excluded since we have no sign control. Finally, the principal symbol is

$$
\lambda_{1}^{+}=\mp \sqrt{-\tau-h^{-2} \xi^{2}+V_{r}} .
$$

The sign of $\lambda_{1}^{+}$is chosen to fix the outgoing wave. The term $-\tau-h^{-2} \xi^{2}+V_{r}$ is real valued and its square-root is a complex number with a null or positive imaginary part. Therefore, the minus sign must be considered to get a negative imaginary part for $\lambda_{1}^{+}$. The principal symbol is then

$$
\lambda_{1}^{+}=-\sqrt{-\tau-h^{-2} \xi^{2}+V_{r}} .
$$

The computation of $\lambda_{0}^{+}$is obtained by considering the first order terms in (71). These are solutions to

$$
i \partial_{r} \lambda_{1}^{+}+i \kappa_{r} \lambda_{1}^{+}+2 \lambda_{1}^{+} \lambda_{0}^{+}=i h^{-1}\left(\partial_{s} h^{-1}\right) \xi
$$

However, since $\lambda_{1}^{+}$is non homogeneous, $\partial_{r} \lambda_{1}^{+}$is the sum of two terms with different homogeneities, respectively 1 and -1 ,

$$
\partial_{r} \lambda_{1}^{+}=\frac{\partial_{r}\left(h^{-1}\right) \xi^{2}}{2 \sqrt{-\tau-h^{-2} \xi^{2}+V_{r}}}-\frac{\partial_{r} V_{r}}{2 \sqrt{-\tau-h^{-2} \xi^{2}+V_{r}}} .
$$

We use the notation: $\left(\partial_{r} \lambda_{1}^{+}\right)_{1}$ to designate the part of $\partial_{r} \lambda_{1}^{+}$which is of order 1 , and similarly for the order -1 . With these notations, the equation for the first order terms writes

$$
i\left(\partial_{r} \lambda_{1}^{+}\right)_{1}+i \kappa_{r} \lambda_{1}^{+}+2 \lambda_{1}^{+} \lambda_{0}^{+}=i h^{-1}\left(\partial_{s} h^{-1}\right) \xi
$$

The term of order $-1\left(\partial_{r} \lambda_{1}^{+}\right)_{-1}$ will be considered when the terms of order -1 will be collected in the computation of $\lambda_{-2}^{+}$. Hence, all the terms of each equation must be carefully studied to know their correct order. Finally, we have

$$
\lambda_{0}^{+}=-\frac{i}{2} \kappa_{r}+\frac{i}{4} \frac{\left(\partial_{r} h^{-2}\right) \xi^{2}}{-\tau-h^{-2} \xi^{2}+i h^{-1}\left(\partial_{s} h^{-1}\right) \xi+V_{r}}-\frac{i}{4} \frac{h^{-2}\left(\partial_{s} h^{-2}\right) \xi^{3}}{{\sqrt{-\tau-h^{-2} \xi^{2}+i h^{-1}\left(\partial_{s} h^{-1}\right) \xi+V_{r}}}^{3}}
$$

We also compute $\lambda_{-1}^{+}$and $\lambda_{-2}^{+}$which are next evaluated at $r=0$ to get the associated operators $\widetilde{\Lambda^{+}}$. 
This gives

$$
\begin{aligned}
\tilde{\lambda}_{1}=- & \sqrt{-\tau-\xi^{2}+V}, \\
\tilde{\lambda}_{0}=- & \frac{i}{2} \kappa-\frac{i}{2} \frac{\kappa \xi^{2}}{-\tau-\xi^{2}+V}, \\
\tilde{\lambda}_{-1}= & -\frac{1}{8} \frac{\kappa^{2}}{\sqrt{-\tau-\xi^{2}+V}}-\frac{1}{2} \frac{\partial_{s} \kappa \xi}{-\tau-\xi^{2}+V}-\frac{3}{4} \frac{\kappa^{2} \xi^{2}}{\sqrt{-\tau-\xi^{2}+V}}-\frac{1}{2} \frac{\partial_{s} \kappa \xi^{3}}{\left(-\tau-\xi^{2}+V\right)^{2}} \\
& -\frac{5}{8} \frac{\kappa^{2} \xi^{4}}{\sqrt{-\tau-\xi^{2}+V}}, \\
& -i \frac{\partial_{\mathbf{n}} V}{-\tau-\xi^{2}+V}+\frac{i}{8} \frac{\partial_{s}^{2} \kappa}{\tau-\xi^{2}+V}+\frac{i}{8} \frac{\kappa^{3}}{\tau-\xi^{2}+V}+\frac{13 i}{8} \frac{\kappa \partial_{s} \kappa \xi}{\sqrt{\tau-\xi^{2}+V}}+\frac{5 i \partial_{s}^{2} \kappa \xi^{4}}{\sqrt{\tau-\xi^{2}+V}} \\
& +\frac{13 i}{8} \frac{\kappa^{3} \xi^{2}}{\left(\tau-\xi^{2}+V\right)^{2}}+\frac{3 i \partial_{s}^{2} \kappa \xi^{2}}{\left(\tau-\xi^{2}+V\right)^{2}}+\frac{19 i \kappa \partial_{s} \kappa \xi^{3}}{\sqrt{\tau-\xi^{2}+V}}+\frac{5 \eta^{5}}{\left(\tau-\xi^{2}+V\right)^{3}} \\
& +\frac{27 i}{8} \frac{\kappa^{3} \xi^{4}}{\left(\tau-\xi^{2}+V\right)^{3}}+\frac{25 i \kappa \partial_{s} \kappa \xi^{5}}{\tilde{\lambda}_{\tau-\xi^{2}+V}^{7}}+\frac{15 i}{8} \frac{\kappa^{3} \xi^{6}}{\left(\tau-\xi^{2}+V\right)^{4}} .
\end{aligned}
$$

For the symbols (74)-(77), we get the $\mathrm{ABC}_{2}^{M}$ of order $M, M \in\{1,2,3,4\}$,

$$
\partial_{\mathbf{n}} u+i \sum_{j=0}^{M-1} O p\left(\widetilde{\lambda}_{1-j}\right) u=0, \quad \text { on } \Sigma_{T} .
$$

The next step consists in interpreting the operators $O p\left(\widetilde{\lambda}_{j}\right)$ in such a way that the $\mathrm{ABC}(54)$ is explicit in terms of operators. As before, we consider both the Taylor and Padé approaches.

\subsection{Interpretation of the ABCs for the Taylor approach}

In this approach, we consider an asymptotic expansion of the symbols $\widetilde{\lambda}_{1}, \widetilde{\lambda}_{0}, \widetilde{\lambda}_{-1}$ and $\tilde{\lambda}_{-2}$ for large $|\tau|$ in the $E$-quasi hyperbolic zone. Taking the notations (55), we get the symbols asymptotic expansions to the order -1 in $\tau$

$$
\begin{aligned}
& \left(\widetilde{\lambda}_{1}\right)_{(-1)}=-\sqrt{-\tau}+\left(\frac{\xi^{2}}{2}-\frac{V}{2}\right) \frac{1}{\sqrt{-\tau}}, \\
& \left(\widetilde{\lambda}_{0}\right)_{(-1)}=-\frac{i}{2} \kappa+\frac{i}{2} \frac{\kappa \xi^{2}}{\tau} \\
& \left(\widetilde{\lambda}_{-1}\right)_{(-1)}=-\frac{1}{8} \frac{\kappa^{2}}{\sqrt{-\tau}}+\frac{1}{2} \frac{\partial_{s} \kappa \xi}{\tau} \\
& \left(\widetilde{\lambda}_{-2}\right)_{(-1)}=\frac{i}{4} \frac{\partial_{\mathbf{n}} V}{\tau}-\frac{i}{8} \frac{\partial_{s}^{2} \kappa}{\tau}-\frac{i}{8} \frac{\kappa^{3}}{\tau} .
\end{aligned}
$$

Then, we can write the fourth order ABC in terms of symbols

$$
\partial_{\mathbf{n}} u+i \sum_{j=0}^{3} O p\left(\left(\widetilde{\lambda}_{1-j}\right)_{(-1)}\right) u=0, \quad \text { on } \Sigma_{T},
$$


which gives

$$
\begin{aligned}
\partial_{\mathbf{n}} u+e^{-i \pi / 4} \partial_{t}^{1 / 2} u+\frac{\kappa}{2} u-e^{i \pi / 4}\left(\frac{\kappa^{2}}{8}+\frac{\Delta_{\Sigma}}{2}+\frac{V}{2}\right) I_{t}^{1 / 2} u & \\
& +i\left(\frac{\partial_{s}\left(\kappa \partial_{s}\right)}{2}+\frac{\kappa^{3}}{8}+\frac{\partial_{s}^{2} \kappa}{8}-\frac{\partial_{\mathbf{n}} V}{4}\right) I_{t} u=0
\end{aligned}
$$

Like for the gauge change method, we try to obtain a symmetrical ABC to get a priori estimates. We symmetrize the two terms $\frac{V}{2}$ and $\frac{\partial_{\mathbf{n}} V}{4}$, by writing

$$
V=\operatorname{sg}(V) \sqrt{|V|} \sqrt{|V|}
$$

and

$$
\partial_{\mathbf{n}} V=\operatorname{sg}\left(\partial_{\mathbf{n}} V\right) \sqrt{\left|\partial_{\mathbf{n}} V\right|} \sqrt{\left|\partial_{\mathbf{n}} V\right|}
$$

The resulting $\mathrm{ABC}$ is denoted by $\mathrm{ABC}_{2, T}^{4}$. We directly have some lower order $\mathrm{ABCs}$. The result is embedded in the following proposition.

Proposition 3. In strategy II (direct method) and by using a Taylor expansion, the ABCs of order $M$ are given by

$$
\partial_{\mathbf{n}} u+\Lambda_{2, T}^{M} u=0, \quad \text { on } \Sigma_{T},
$$

where the operators $\Lambda_{2, T}^{M}$ are defined on $\Sigma_{T}$ by

$$
\begin{aligned}
& \Lambda_{2, T}^{1} u=e^{-i \pi / 4} \partial_{t}^{1 / 2} u, \\
& \Lambda_{2, T}^{2} u=\Lambda_{2, T}^{1} u+\frac{\kappa}{2} u, \\
& \Lambda_{2, T}^{3} u=\Lambda_{2, T}^{2} u-e^{i \pi / 4}\left(\frac{\kappa^{2}}{8}+\frac{\Delta_{\Sigma}}{2}\right) I_{t}^{1 / 2} u-e^{i \pi / 4} \frac{\operatorname{sg}(V)}{2} \sqrt{|V|} I_{t}^{1 / 2}(\sqrt{|V|} u), \\
& \Lambda_{2, T}^{4} u=\Lambda_{2, T}^{3} u+i\left(\frac{\partial_{s}\left(\kappa \partial_{s}\right)}{2}+\frac{\kappa^{3}+\partial_{s}^{2} \kappa}{8}\right) I_{t} u-i \frac{\operatorname{sg}\left(\partial_{\mathbf{n}} V\right)}{4} \sqrt{\left|\partial_{\mathbf{n}} V\right|} I_{t}\left(\sqrt{\left|\partial_{\mathbf{n}} V\right|} u\right) .
\end{aligned}
$$

The boundary conditions are denoted by $A B C_{2, T}^{M}$ in the sequel.

Remark 4. Let us note the symmetrical form of $\Lambda_{2, T}^{3}$ and $\Lambda_{2, T}^{4}$. We also obtain here the ABCs expected from strategy zero (see subsection 4.2, Remark 1). This shows that strategy II is more general.

\subsection{Interpretation of the ABCs and Padé approach}

For the Padé approach, the first order ABC is obtained by retaining the first symbol $\widetilde{\lambda}_{1}$

$$
\partial_{\mathbf{n}} u-i O p\left(\sqrt{-\tau-\xi^{2}+V}\right) u=0
$$

and the second order $\mathrm{ABC}$ by adding $\widetilde{\lambda}_{0}$

$$
\partial_{\mathbf{n}} u-i O p\left(\sqrt{-\tau-\xi^{2}+V}\right) u+\frac{\kappa}{2} u+\frac{1}{2} O p\left(\frac{\kappa \xi^{2}}{-\tau-\xi^{2}+V}\right) u=0 .
$$

Since we consider the symbols globally, we retain less terms in the expansion. For implementation issues, it is necessary to interpret the operators. We use the following approximations. 
Lemma 3. We have the following equivalences

$$
O p\left(\sqrt{-\tau-\xi^{2}+V}\right) \sim \sqrt{i \partial_{t}+\Delta_{\Sigma}+V} \quad \bmod O P S_{E}^{-1}
$$

and

$$
O p\left(\frac{\kappa \xi^{2}}{-\tau-\xi^{2}+V}\right) \sim-\kappa\left(i \partial_{t}+\Delta_{\Sigma}+V\right)^{-1} \Delta_{\Sigma} \quad \bmod O P S_{E}^{-3}
$$

Proof. Let us consider the first equivalence (90). We set $A=\sqrt{i \partial_{t}+\Delta_{\Sigma}+V}$ which is a first order operator in $\left(\xi^{2}, \tau\right)$. Therefore, it admits the asymptotic expansion: $\sigma(A) \sim \sum_{j=0}^{+\infty} \sigma_{1-j}$, where the symbols $\sigma_{1-j}$ are homogeneous of order $1-j, j \in \mathbb{Z}$. We now determine this asymptotics thanks to

$$
A^{2}=i \partial_{t}+\Delta_{\Sigma}+V=O p\left(-\tau-\xi^{2}+V\right) .
$$

Furthermore, we have

$$
\sigma\left(A^{2}\right)=\sum_{|\alpha|=0}^{+\infty} \frac{(-i)^{|\alpha|}}{\alpha !} \partial_{(\xi, \tau)}^{\alpha} \sigma(A) \partial_{(s, t)}^{\alpha} \sigma(A) .
$$

Using (92) and (93), we compute the symbols $\sigma_{1-j}, j \in \mathbb{Z}$. The identification of the second order terms leads to $-\tau-\xi^{2}+V=\sigma_{1}^{2}$, and to the principal symbol: $\sigma_{1}=\sqrt{-\tau-\xi^{2}+V}$. The identification of the terms of order 1 gives $0=\sigma_{1} \sigma_{0}+\sigma_{0} \sigma_{1}$, and $\sigma_{0}=0$. Similarly, we have: $0=2 \sigma_{1} \sigma_{-1}+\sigma_{0}^{2}-i \partial_{\xi} \sigma_{1} \partial_{s} \sigma_{1}$ and hence $\sigma_{-1}=0$. Finally, the terms of order -1 lead to $2 \sigma_{1} \sigma_{-2}=i \partial_{\xi} \sigma_{1} \partial_{s} \sigma_{1}$, which gives

$$
\sigma_{-2}=-\frac{i}{4} \frac{\left(\partial_{s} V\right) \xi}{\sqrt{-\tau-\xi^{2}+V^{3}}} .
$$

Finally, the asymptotics of $A$ is

$$
\sigma\left(\sqrt{i \partial_{t}+\Delta_{\Sigma}+V}\right) \sim \sqrt{-\tau-\xi^{2}+V}-\frac{i}{4} \frac{\xi \partial_{s} V}{\sqrt{-\tau-\xi^{2}+V}}+\ldots
$$

We deduce that $\sqrt{-\tau-\xi^{2}+V}$ is the principal symbol of $A$ and we have

$$
\sigma\left(\sqrt{i \partial_{t}+\Delta_{\Sigma}+V}\right)=\sqrt{-\tau-\xi^{2}+V} \bmod \mathrm{S}_{E}^{-1}
$$

and (90) at the operators level.

We proceed in a similar way to show (91) by introducing $B=\left(i \partial_{t}+\Delta_{\Sigma}+V\right)^{-1}$. This is an operator of order -2 with a total symbol such that: $\sigma(B)=\sum_{j=0}^{+\infty} \sigma_{-2-j}$. Since $\sigma\left(B^{-1}\right)=\sigma\left(i \partial_{t}+\right.$ $\left.\Delta_{\Sigma}+V\right)=-\tau-\xi^{2}+V$, we have the identity

$$
1=\sigma\left(B B^{-1}\right)=\sum_{|\alpha|=0}^{+\infty} \frac{(-i)^{|\alpha|}}{\alpha !} \partial_{(\xi, \tau)}^{\alpha} \sigma(B) \partial_{(s, t)}^{\alpha} \sigma\left(B^{-1}\right) .
$$

The terms of order 0 give $1=\sigma_{-2} \sigma\left(B^{-1}\right)=\sigma_{-2}\left(-\tau-\xi^{2}+V\right)$ and

$$
\sigma_{-2}=\frac{1}{-\tau-\xi^{2}+V}
$$


We next obtain the equations $0=\sigma_{-3} \sigma\left(B^{-1}\right)$ and $\sigma_{-3}=0$, then $0=\sigma_{-4} \sigma\left(B^{-1}\right)$, and $\sigma_{-4}=0$. Finally, the terms of order -3 lead to $\sigma_{-5} \sigma\left(B^{-1}\right)=i \partial_{\xi} \sigma_{-2} \partial_{s} \sigma\left(B^{-1}\right)$, and

$$
\sigma_{-5}=\frac{2 i \partial_{s} V \xi}{\left(-\tau-\xi^{2}+V\right)^{3}} .
$$

We deduce the asymptotics of the inverse operator

$$
\sigma\left(\left(i \partial_{t}+\Delta_{\Sigma}+V\right)^{-1}\right) \sim \frac{1}{-\tau-\xi^{2}+V}+\frac{2 i \partial_{s} V \xi}{\left(-\tau-\xi^{2}+V\right)^{3}}+\ldots
$$

We can now compute the asymptotics of $-\kappa\left(i \partial_{t}+\Delta_{\Sigma}+V\right)^{-1} \Delta_{\Sigma}$. Function $\kappa$ is independent of $\xi$ and $\tau$, and so

$$
\sigma\left(-\kappa\left(i \partial_{t}+\Delta_{\Sigma}+V\right)^{-1} \Delta_{\Sigma}\right)=-\kappa \sigma\left(\left(i \partial_{t}+\Delta_{\Sigma}+V\right)^{-1} \Delta_{\Sigma}\right)
$$

By the composition formula, we can write that

$$
\begin{aligned}
-\kappa \sigma\left(\left(i \partial_{t}+\Delta_{\Sigma}+V\right)^{-1} \Delta_{\Sigma}\right) & =-\kappa \sum_{|\alpha|=0}^{+\infty} \frac{(-i)^{|\alpha|}}{\alpha !} \partial_{(\xi, \tau)}^{\alpha} \sigma\left(\left(i \partial_{t}+\Delta_{\Sigma}+V\right)^{-1}\right) \partial_{(s, t)}^{\alpha} \sigma\left(\Delta_{\Sigma}\right) \\
& =\kappa \xi^{2} \sigma\left(\left(i \partial_{t}+\Delta_{\Sigma}+V\right)^{-1}\right)
\end{aligned}
$$

since all the terms are equal to zero for $|\alpha| \geq 1$. Hence, we have

$$
\sigma\left(-\kappa\left(i \partial_{t}+\Delta_{\Sigma}+V\right)^{-1} \Delta_{\Sigma}\right) \sim \frac{\kappa \xi^{2}}{-\tau-\xi^{2}+V}+\frac{2 i \kappa \partial_{s} V \xi^{3}}{\left(-\tau-\xi^{2}+V\right)^{3}}+\ldots
$$

The symbol $\frac{\kappa \xi^{2}}{-\tau-\xi^{2}+V}$ can finally be interpreted as the principal symbol of $-\kappa\left(i \partial_{t}+\Delta_{\Sigma}+V\right)^{-1} \Delta_{\Sigma}$, leading to (91).

From Lemma 3, we deduce $\mathrm{ABC}$ for strategy II that we denote by $\mathrm{ABC}_{2, P}^{M}$, and which are given in Proposition 4.

Proposition 4. In strategy II and following a Padé approach, the ABCs of order $M$ are given by

$$
\partial_{\mathbf{n}} u+\Lambda_{2, P}^{M} u=0, \quad \text { on } \Sigma_{T},
$$

where the operators $\Lambda_{2, P}^{M}$ are defined on $\Sigma_{T}$ by

$$
\begin{aligned}
& \Lambda_{2, P}^{1} u=-i \sqrt{i \partial_{t}+\Delta_{\Sigma}+V} u, \\
& \Lambda_{2, P}^{2} u=\Lambda_{2, P}^{2} u+\frac{\kappa}{2} u-\frac{\kappa}{2}\left(i \partial_{t}+\Delta_{\Sigma}+V\right)^{-1} \Delta_{\Sigma} u .
\end{aligned}
$$

The $A B C$ s are denoted by $A B C_{2, P}^{M}$.

Remark 5. We saw in paragraph 3.2 that for a time independent radial potential we have the correspondence between the gauge change and direct methods. It can be proved that this is the case for the $\mathrm{ABCs} \mathrm{ABC}_{1, P}^{M}$ and $\mathrm{ABC}_{2, P}^{M}$ by using the Padé approach while not for the Taylor approach where the symmetry is broken. 


\section{$7 \quad$ A priori estimates}

\subsection{Principle}

We have four families of ABCs related to Strategy I and II, Taylor or Padé approximation. These ABCs are given in Propositions 1, 2, 3 and 4 for $\mathrm{ABC}_{1, T}^{M}, \mathrm{ABC}_{1, P}^{M}, \mathrm{ABC}_{2, T}^{M}$ and $\mathrm{ABC}_{2, P}^{M}$, respectively. In [7], for the free-potential equation, $\mathrm{ABCs}$ have been built and correspond to strategy II with Taylor approximation for $V=0$. For these ABCs, we have the following result [7].

Proposition 5. Let $u_{0} \in L^{2}(\Omega)$ and $u$ solution to

$$
\left\{\begin{array}{l}
\left(i \partial_{t}+\Delta u\right)=0, \quad \text { in } \Omega \times \mathbb{R}^{+*}, \\
\partial_{\mathbf{n}} u+T^{M} u=0, \quad \text { on } \Sigma \times \mathbb{R}^{+*}, \\
u(x, 0)=u_{0}(x), \quad \text { in } \Omega,
\end{array}\right.
$$

where the operators $T^{M}$ are defined on $\Sigma \times \mathbb{R}^{+*}$ by

$$
\begin{aligned}
& T^{1} u=e^{-i \pi / 4} \partial_{t}^{1 / 2} u, \quad T^{2} u=T^{1} u+\frac{\kappa}{2} u, \\
& T^{3} u=T^{2} u-e^{i \pi / 4}\left(\frac{\kappa^{2}}{8}+\frac{\Delta_{\Sigma}}{2}\right) I_{t}^{1 / 2} u, \\
& T^{4} u=T^{3} u+i\left(\frac{\kappa^{3}}{8}+\frac{1}{2} \partial_{s}\left(\kappa \partial_{s}\right)+\frac{\partial_{s}^{2} \kappa}{8}\right) I_{t} u .
\end{aligned}
$$

Then, $u$ is unique and defined in $C\left([0 ; T], L^{2}(\Omega)\right)$. Moreover, u satisfies the energy inequality

$$
\forall t \in[0 ; T], \quad\|u(t)\|_{L^{2}(\Omega)} \leq\left\|u_{0}\right\|_{L^{2}(\Omega)} .
$$

The idea is to generalize this Proposition to the $\mathrm{ABC}$ with potential, that is $\mathrm{ABC}_{1, T}^{M}$ or $\mathrm{ABC}_{2, T}^{M}$. For $\Lambda_{1, T}^{M}$ and $\Lambda_{2, T}^{M}$, this seems pretty simple to generalize Proposition 5 to $\Lambda_{1, T}^{2}$ because of the symmetry of the operators. This is however more difficult for $\Lambda_{1, T}^{3}$ (and $\Lambda_{1, T}^{4}$ ) since we have no sign control on $i \partial_{s}^{2} \mathcal{V}-\left(\partial_{s} \mathcal{V}\right)^{2}$.

For the second strategy, the operators are closer to $T^{M}$ since there is no change of variable. The interesting point is to analyze if the added terms modify or not the result. For $\Lambda_{2, T}^{1}=T^{1}$ and

$\Lambda_{2, T}^{2}=T^{2}$, the result is direct since it does not include any potential term. The operators $\Lambda_{2, T}^{3}$ and $\Lambda_{2, T}^{4}$ are respectively modified by terms $V$ and $\partial_{\mathbf{n}} V$ compared to $T^{3}$ and $T^{4}$. Our study will mainly analyze these two additional terms. We will need the following Lemma [9].

Lemma 4. Let $\varphi \in H^{1 / 4}\left([0 ; t], L^{2}(\Sigma)\right)$ and $\psi \in L^{2}\left([0 ; t], L^{2}(\Sigma)\right)$ that we extend to zero on $\mathbb{R} \backslash[0 ; t]$. Then we have

$$
\begin{aligned}
& \int_{\mathbb{R} \times \Sigma} \overline{\varphi\left(s, t^{\prime}\right)} \partial_{t^{\prime}}^{1 / 2} \varphi\left(s, t^{\prime}\right) d t^{\prime} d \Sigma \quad \in e^{i \pi / 4} \mathbb{R}^{+} \cup e^{-i \pi / 4} \mathbb{R}^{+}, \\
& \int_{\mathbb{R} \times \Sigma} \overline{\psi\left(s, t^{\prime}\right)} I_{t^{\prime}}^{1 / 2} \psi\left(s, t^{\prime}\right) d t^{\prime} d \Sigma \quad \in e^{i \pi / 4} \mathbb{R}^{+} \cup e^{-i \pi / 4} \mathbb{R}^{+}, \\
& \int_{\mathbb{R} \times \Sigma} \overline{\psi\left(s, t^{\prime}\right)} I_{t^{\prime}} \psi\left(s, t^{\prime}\right) d t^{\prime} d \Sigma \quad \in i \mathbb{R} .
\end{aligned}
$$


Proof. Let $\tau$ and $\xi$ be respectively the Fourier covariables of $t^{\prime}$ and $s$ and let $\hat{u}$ be the Fourier transform of $u$. Let us first remark that for a general pseudodifferential operator $P\left(s, t^{\prime}, \xi, \tau\right)$ with symbol $p\left(s, t^{\prime}, \xi, \tau\right)$, we have the identity

$$
\int_{\mathbb{R} \times \Sigma} \overline{\varphi\left(s, t^{\prime}\right)} P\left(s, t^{\prime}, \partial_{s}, \partial_{t}\right) \varphi\left(s, t^{\prime}\right) d t^{\prime} d \Sigma=\int_{\mathbb{R} \times \mathbb{R}} p\left(s, t^{\prime}, \xi, \tau\right)|\hat{\varphi}(\xi, \tau)|^{2} d \tau d \xi .
$$

Indeed, Plancherel's Theorem implies that

$$
\int_{\mathbb{R} \times \Sigma} \overline{\varphi\left(s, t^{\prime}\right)} P \varphi\left(s, t^{\prime}\right) d t^{\prime} d \Sigma=\int_{\mathbb{R} \times \mathbb{R}} \overline{\mathscr{F}(\varphi)(\xi, \tau)} \mathscr{F}\left(P \varphi\left(s, t^{\prime}\right)\right)(\xi, \tau) d \tau d \xi .
$$

But, from the definition of the symbol of an operator, we have

$$
\mathscr{F}\left(P \varphi\left(s, t^{\prime}\right)\right)(\xi, \tau)=\sigma(P) \hat{\varphi}(\xi, \tau)=p\left(s, t^{\prime}, \xi, \tau\right) \hat{\varphi}(\xi, \tau)
$$

and so (102)

$$
\int_{\mathbb{R} \times \Sigma} \overline{\varphi\left(s, t^{\prime}\right)} P \varphi\left(s, t^{\prime}\right) d t^{\prime} d \Sigma=\int_{\mathbb{R} \times \mathbb{R}} \overline{\hat{\varphi}(\xi, \tau)} p\left(s, t^{\prime}, \xi, \tau\right) \hat{\varphi}(\xi, \tau) d t^{\prime} d \Sigma=\int_{\mathbb{R} \times \mathbb{R}} p\left(s, t^{\prime}, \xi, \tau\right)|\hat{\varphi}(\xi, \tau)|^{2} d \tau d \xi .
$$

We next apply this result to $\partial_{t^{\prime}}^{1 / 2}$ with symbol $\sqrt{i \tau}$. We must determine the part of the complex plane in which this symbol lies. For positive $\tau$ we have $\sqrt{i \tau}=e^{i \pi / 4} \sqrt{\tau}$, and for negative $\tau, \sqrt{i \tau}=e^{-i \pi / 4} \sqrt{-\tau}$, leading to the expected property (99). A very similar proof gives the result for $I_{t^{\prime}}^{1 / 2}$ et $I_{t^{\prime}}$ with respective symbols $\sigma\left(I_{t^{\prime}}^{1 / 2}\right)=\frac{1}{\sqrt{i \tau}}$ and $\sigma\left(I_{t^{\prime}}\right)=\frac{1}{i \tau}$.

Lemma 4 is the basic ingredient to derive a priori estimates for $\mathrm{ABC}_{1, T}^{M}$ and $\mathrm{ABC}_{2, T}^{M}$.

Remark 6. The Padé based ABCs will not be considered here. We cannot apply our proof and more developments should be considered.

\subsection{A priori estimates for $\mathrm{ABC}_{2, T}^{M}$}

Concerning the family of $\mathrm{ABCs} \mathrm{ABC}_{2, T}^{M}$, the following result holds.

Theorem 2. Let $u_{0} \in L^{2}(\Omega)$ an initial data with compact support in $\Omega$. Let $V \in C^{\infty}\left(\mathbb{R}^{2} \times \mathbb{R}^{+}, \mathbb{R}\right)$ be a real-valued potential. We denote by $u$ a solution to the initial boundary value problem

$$
\left\{\begin{array}{l}
i \partial_{t} u+\Delta u+V u=0, \quad \text { in } \Omega_{T} \\
\partial_{\mathbf{n}} u+\Lambda_{2, T}^{M} u=0, \quad \text { on } \Sigma_{T} \\
u(x, 0)=u_{0}(x), \quad \text { in } \Omega
\end{array}\right.
$$

where the operators $\Lambda_{2, T}^{M}, M \in\{1,2,3,4\}$, are defined in Proposition 3. Then, u satisfies the inequality

$$
\forall t>0, \quad\|u(t)\|_{L^{2}(\Omega)} \leq\left\|u_{0}\right\|_{L^{2}(\Omega)}
$$

for $M=1$ and $M=2$. Moreover, this inequality is also satisfied for $M=3$ under the assumption that $V$ is positive on $\Sigma$, and for $M=4$ under the hypothesis that $\operatorname{sg}\left(\partial_{\mathbf{n}} V\right)$ is constant over $\Sigma$ for any time. In particular, this implies the uniqueness of the solution u of the initial boundary value problem (103). 
Proof. Let $\tilde{T} \in] 0 ; T]$. Let us introduce $\mathbf{x}=(x, y) \in \mathbb{R}^{2}$. We begin by extending $u$ to a function $\tilde{u}$ defined over $\mathbb{R}$ in the following way

$$
\tilde{u}(\cdot, t)= \begin{cases}u(\cdot, t) & \text { for } t \in[0 ; \tilde{T}] \\ 0 & \text { for } t \in]-\infty ; 0[\cup] \tilde{T} ;+\infty[.\end{cases}
$$

In the distributions sense, we obtain

$$
\begin{aligned}
& \partial_{t} \tilde{u}(\mathbf{x}, t)= \begin{cases}\partial_{t} u(\mathbf{x}, t)-u(\mathbf{x}, \tilde{T}) \delta_{\tilde{T}}(t)+u_{0}(\mathbf{x}) \delta_{0}(t), & t \in[0 ; \tilde{T}] \\
0, & t \in]-\infty ; 0[\cup] \tilde{T} ;+\infty[\end{cases} \\
& \Delta \tilde{u}(\mathbf{x}, t)= \begin{cases}\Delta u(\mathbf{x}, t), & t \in[0 ; \tilde{T}] \\
0, & t \in]-\infty ; 0[\cup] \tilde{T} ;+\infty[\end{cases} \\
& \partial_{\mathbf{n}} \tilde{u}+\Lambda_{2, T}^{M} \tilde{u}=0 .
\end{aligned}
$$

The function $\tilde{u}$ satisfies the equation

$$
i \partial_{t} \tilde{u}+\Delta \tilde{u}+V \tilde{u}=-i u(\mathbf{x}, \tilde{T}) \delta_{\tilde{T}}+i u_{0}(\mathbf{x}) \delta_{0}(t), \quad \text { on } \Omega \times \mathbb{R}
$$

Multiplying this equation by $-i \overline{\tilde{u}(\mathbf{x}, t)}$, we get

$$
\overline{\tilde{u}} \partial_{t} \tilde{u}-i \overline{\tilde{u}} \Delta \tilde{u}-i \overline{\tilde{u}} V \tilde{u}=-u(\mathbf{x}, \tilde{T}) \overline{\tilde{u}} \delta_{\tilde{T}}(t)+u_{0}(\mathbf{x}) \overline{\tilde{u}} \delta_{0}(t) .
$$

We integrate by part over $\Omega$

$$
\begin{aligned}
\int_{\Omega} \overline{\tilde{u}} \partial_{t} \tilde{u} d \Omega-i \int_{\Sigma} \overline{\tilde{u}} \partial_{\mathbf{n}} \tilde{u} d \Sigma+i \int_{\Omega}|\nabla \tilde{u}|^{2} d \Omega-i \int_{\Omega} V|\tilde{u}|^{2} d \Omega & \\
& =-\int_{\Omega} u(\mathbf{x}, \tilde{T}) \overline{\tilde{u}} \delta_{\tilde{T}}(t) d \Omega+\int_{\Omega} u_{0}(\mathbf{x}) \overline{\tilde{u}} \delta_{0}(t) d \Omega
\end{aligned}
$$

and next take the real part of this expression by assuming that $V$ is a real-valued potential

$$
\int_{\Omega} \partial_{t} \frac{|\tilde{u}|^{2}}{2} d \Omega-\operatorname{Re}\left(i \int_{\Sigma} \partial_{\mathbf{n}} \tilde{u} \tilde{u} d \Sigma\right)=-\operatorname{Re}\left(\int_{\Omega} u(\mathbf{x}, \tilde{T}) \overline{\tilde{u}} \delta_{\tilde{T}}(t) d \Omega\right)+\operatorname{Re}\left(\int_{\Omega} u_{0}(\mathbf{x}) \overline{\tilde{u}} \delta_{0}(t) d \Omega\right)
$$

We integrate on $t \in \mathbb{R}$. The terms in the left hand side give

$$
\int_{\mathbb{R}} \int_{\Omega} u(\mathbf{x}, \tilde{T}) \overline{\tilde{u}(\mathbf{x}, t)} \delta_{\tilde{T}}(t) d \Omega d t=\int_{\Omega} u(\mathbf{x}, \tilde{T}) \int_{\mathbb{R}} \overline{\tilde{u}(\mathbf{x}, t)} \delta_{\tilde{T}}(t) d t d \Omega=\int_{\Omega} u(\mathbf{x}, \tilde{T}) \overline{\tilde{u}(\mathbf{x}, \tilde{T})} d \Omega=\|u(\tilde{T})\|_{L^{2}(\Omega)}^{2}
$$

since $\tilde{u}(\mathbf{x}, \tilde{T})=u(\mathbf{x}, \tilde{T})$, and

$$
\int_{\mathbb{R}} \int_{\Omega} u_{0}(\mathbf{x}) \overline{\tilde{u}(\mathbf{x}, t)} \delta_{0}(t) d \Omega d t=\int_{\Omega} u_{0}(\mathbf{x}) \overline{\tilde{u}(\mathbf{x}, 0)} d \Omega=\left\|u_{0}\right\|_{L^{2}(\Omega)}^{2},
$$

because $\tilde{u}(\mathbf{x}, 0)=u(\mathbf{x}, 0)=u_{0}(\mathbf{x})$. Furthermore, since $\tilde{u}$ is zero at $\pm \infty$, we have

$$
\int_{\Omega} \int_{\mathbb{R}} \partial_{t} \frac{|\tilde{u}|^{2}}{2} d t d \Omega=\int_{\Omega}\left[\frac{|\tilde{u}(\mathbf{x}, t)|^{2}}{2}\right]_{-\infty}^{+\infty}=0
$$


Finally, after a time integration of (106), we obtain

$$
\operatorname{Re}\left(i \int_{\Sigma \times \mathbb{R}} \overline{\tilde{u}} \partial_{\mathbf{n}} \tilde{u} d \Sigma d t\right)=\|u(\tilde{T})\|_{L^{2}(\Omega)}^{2}-\left\|u_{0}\right\|_{L^{2}(\Omega)}^{2}
$$

for any $\tilde{T} \in[0 ; T]$, and where $\partial_{\mathbf{n}} \tilde{u}$ is given by the chosen boundary condition. For the sake of conciseness, we note $u$ instead of $\tilde{u}$, having in mind that $u$ is equal to zero outside $[0 ; \tilde{T}]$.

Let us first begin by $\mathrm{ABC}_{2, T}^{2}$ given by the operator (85). We have to analyze the sign of the real part of the two following terms

$$
\int_{\Sigma \times \mathbb{R}} i \bar{u} \partial_{\mathbf{n}} u d \Sigma d t=-i e^{-i \pi / 4} \int_{\Sigma \times \mathbb{R}} \bar{u} \partial_{t}^{1 / 2} u d \Sigma d t-\frac{i}{2} \int_{\Sigma \times \mathbb{R}} \kappa|u|^{2} d \Sigma d t .
$$

To this end we use the identity (102). We have

$$
-i e^{-i \pi / 4} \int_{\Sigma \times \mathbb{R}} \bar{u} \partial_{t}^{1 / 2} u d \Sigma d t=-i e^{-i \pi / 4} \int_{\mathbb{R} \times \mathbb{R}}|\hat{u}(\xi, \tau)|^{2} \sigma\left(\partial_{t}^{1 / 2}\right) d \xi d \tau .
$$

However, $\sigma\left(\partial_{t}^{1 / 2}\right)=\sqrt{i \tau}$ which varies in $e^{-i \pi / 4} \mathbb{R}^{+} \cup e^{i \pi / 4} \mathbb{R}^{+}$for $\tau$ in $\mathbb{R}$, implies that the real part of (108) is negative. Since the second term is purely imaginary, (104) is proved for the second order $\mathrm{ABC}$.

We consider now the fourth order $\mathrm{ABC} \mathrm{ABC}_{2, T}^{4}$ given by the operator (87). The boundary condition can be decomposed in seven terms $A_{j}, 1 \leq j \leq 7$, as follows

$$
\begin{aligned}
\int_{\Sigma \times \mathbb{R}} i \bar{u} \partial_{\mathbf{n}} u d \Sigma d t= & \int_{\Sigma \times \mathbb{R}}\left(-i e^{-i \pi / 4} \bar{u} \partial_{t}^{1 / 2} u+i e^{i \pi / 4} \bar{u} \frac{\Delta_{\Sigma}}{2} I_{t}^{1 / 2} u\right) d \Sigma d t-\frac{i}{2} \int_{\Sigma \times \mathbb{R}} \kappa|u|^{2} d \Sigma d t \\
& +i e^{i \pi / 4} \int_{\Sigma \times \mathbb{R}} \frac{\kappa^{2}}{8} \bar{u} I_{t}^{1 / 2} u d \Sigma d t+i e^{i \pi / 4} \int_{\Sigma \times \mathbb{R}} \frac{\operatorname{sg}(V)}{2} \sqrt{|V|} \bar{u} I_{t}^{1 / 2}(\sqrt{|V|} u) d \Sigma d t \\
& -\frac{1}{2} \int_{\Sigma \times \mathbb{R}} \bar{u} \partial_{s}\left(\kappa \partial_{s}\right) I_{t} u d t \Sigma d t+\int_{\Sigma \times \mathbb{R}} \frac{\kappa^{3}+\partial_{s}^{2} \kappa}{8} \bar{u} I_{t} u d \Sigma d t \\
& -\int_{\Sigma \times \mathbb{R}} \frac{\operatorname{sg}\left(\partial_{\mathbf{n}} V\right)}{4} \sqrt{\left|\partial_{\mathbf{n}} V\right| \bar{u}} I_{t}\left(\sqrt{\left|\partial_{\mathbf{n}} V\right|} u\right) d \Sigma d t \\
= & \sum_{j=1}^{7} A_{j} .
\end{aligned}
$$

Therefore, we have to study the sign of the real part of each term. We use several times the identity (102). We have

$$
A_{1}=\int_{\mathbb{R} \times \mathbb{R}}|\hat{u}(\xi, \tau)|^{2} \sigma\left(-i e^{-i \pi / 4} \partial_{t}^{1 / 2}+i e^{i \pi / 4} \frac{\Delta_{\Sigma}}{2} I_{t}^{1 / 2}\right) d \xi d \tau
$$

Furthermore, we also can write that

$$
a_{1}(\xi, \tau):=\sigma\left(-i e^{-i \pi / 4} \partial_{t}^{1 / 2}+i e^{i \pi / 4} \frac{\Delta_{\Sigma}}{2} I_{t}^{1 / 2}\right)=\frac{-i}{2}\left(2 e^{-i \pi / 4} \sqrt{i \tau}+e^{i \pi / 4} \frac{\xi^{2}}{\sqrt{i \tau}}\right) .
$$


For $\tau>0$, we have $\sqrt{i \tau}=e^{i \pi / 4} \sqrt{\tau}$ and so

$$
a_{1}(\xi, \tau)=\frac{-i}{2}\left(2 \sqrt{\tau}+\frac{\xi^{2}}{\sqrt{\tau}}\right)
$$

The symbol $a_{1}(\xi, \tau)$ is then a pure imaginary number in this case. For $\tau<0$, we write $\sqrt{i \tau}=$ $e^{-i \pi / 4} \sqrt{-\tau}$ and we have

$$
a_{1}(\xi, \tau)=\frac{-i}{2}\left(-2 i \sqrt{-\tau}+i \frac{\xi^{2}}{\sqrt{-\tau}}\right)=-\sqrt{-\tau}+\frac{1}{2} \frac{\xi^{2}}{\sqrt{-\tau}}=\frac{1}{2 \sqrt{-\tau}}\left(\tau+\tau+\xi^{2}\right) .
$$

However, we work in the $E$-quasi hyperbolic zone $\mathcal{H}$ in which $\tau+\xi^{2}<0$. As a consequence, the real number $a_{1}(\xi, \tau)$ satisfies $a_{1}(\xi, \tau)<\frac{\tau}{2 \sqrt{-\tau}}<0$ and its real part is negative. Finally, we have $\operatorname{Re}\left(A_{1}\right) \leq 0$. The term $A_{2}$ is purely imaginary. The term $A_{3}$ fulfills

$$
A_{3}=i e^{i \pi / 4} \int_{\mathbb{R} \times \mathbb{R}}|\hat{u}(\xi, \tau)|^{2} a_{3}(\xi, \tau) d \xi d \tau,
$$

where

$$
a_{3}(\xi, \tau)=\sigma\left(\frac{\kappa^{2}}{8} I_{t}^{1 / 2}\right)=\frac{\kappa^{2}}{8} \frac{1}{\sqrt{i \tau}} \in e^{i \pi / 4} \mathbb{R}^{+} \cup e^{-i \pi / 4} \mathbb{R}^{+} .
$$

We deduce that $A_{3}$ varies in $\mathbb{R}^{-} \cup i \mathbb{R}^{+}$and so has a real negative part. Since we assume that $V$ remains positive over $\Sigma$, and then $\operatorname{sg}(V)$ is equal to 1 , we can write $A_{4}$ by applying the Plancherel's identity in time

$$
\begin{aligned}
A_{4} & =i e^{i \pi / 4} \int_{\Sigma} \frac{1}{2} \int_{\mathbb{R}} \sqrt{|V|} \bar{u} I_{t}^{1 / 2}(\sqrt{|V|} u) d t d \Sigma, \\
& =i e^{i \pi / 4} \int_{\Sigma} \frac{1}{2} \int_{\mathbb{R}}\left|\mathscr{F}_{t}(\sqrt{|V|} u)\right|^{2}(s, \tau) \frac{1}{\sqrt{i \tau}} d \tau d \Sigma .
\end{aligned}
$$

Here, we apply Lemma 4 but only working on the time integral and taking the time Fourier transform. The integral over $\tau$ varies in $e^{i \pi / 4} \mathbb{R}^{+} \cup e^{-i \pi / 4} \mathbb{R}^{+}$, and so $A_{4} \in \mathbb{R}^{-} \cup i \mathbb{R}^{+}$and $A_{4}$ has a negative real part.

The next three additional terms are related to the fourth order ABC. Under the assumption on $\partial_{\mathbf{n}} V$, we show that they are all purely imaginary. The first one, $A_{5}$, is treated by a integration by part over the closed curve $\Sigma$ and by using the commutativity of $I_{t}$ and $\partial_{s}$

$$
\int_{\Sigma} \bar{u} \partial_{s}\left(\kappa \partial_{s} I_{t} u\right) d \Sigma=-\int_{\Sigma} \partial_{s} \bar{u} \kappa \partial_{s} I_{t} u d \Sigma=-\int_{\Sigma} \partial_{s} \bar{u} \kappa I_{t}\left(\partial_{s} u\right) d \Sigma
$$

and then

$$
A_{5}=\frac{1}{2} \int_{\Sigma \times \mathbb{R}} \overline{\partial_{s} u} \kappa I_{t}\left(\partial_{s} u\right) d \Sigma d t=\frac{1}{2} \int_{\mathbb{R} \times \mathbb{R}}\left|\widehat{\partial_{s} u}(\xi, \tau)\right|^{2} \frac{\kappa}{i \tau} d \xi d \tau .
$$

Since $\kappa$ is a real number, the term $A_{5}$ is a pure imaginary number. The term $A_{6}$ is also purely imaginary since

$$
A_{6}=\int_{\mathbb{R} \times \mathbb{R}}|\hat{u}(\xi, \tau)|^{2} \frac{\kappa^{3}+\partial_{s}^{2} \kappa}{8} \frac{1}{i \tau} d \xi d \tau .
$$


Finally, for $A_{7}$ we consider the time integral

$$
\begin{aligned}
A_{7} & =-\int_{\Sigma} \frac{\operatorname{sg}\left(\partial_{\mathbf{n}} V\right)}{4} \int_{\mathbb{R}} \sqrt{\left|\partial_{\mathbf{n}} V\right|} \bar{u} I_{t}\left(\sqrt{\left|\partial_{\mathbf{n}} V\right|} u\right) d t d \Sigma \\
& =-\int_{\Sigma} \frac{\operatorname{sg}\left(\partial_{\mathbf{n}} V\right)}{4} \int_{\mathbb{R}}\left|\mathscr{F}_{t}\left(\sqrt{\left|\partial_{\mathbf{n}} V\right|} u\right)(s, \tau)\right|^{2} \frac{1}{i \tau} d \tau d \Sigma
\end{aligned}
$$

which is purely imaginary. Finally, we have

$$
\operatorname{Re}\left(i \int_{\Sigma \times \mathbb{R}} \overline{\tilde{u}} \partial_{\mathbf{n}} \tilde{u} d \Sigma d t\right) \leq 0
$$

and then, for any time $\tilde{T} \in[0 ; T],\|u(\tilde{T})\|_{L^{2}(\Omega)} \leq\left\|u_{0}\right\|_{L^{2}(\Omega)}$, ending hence the proof for $M=4$.

To get the result for the third order $\mathrm{ABC}$, we do not consider $A_{5}, A_{6}$ and $A_{7}$.

\subsection{A priori estimates for $\mathrm{ABC}_{1, T}^{M}$}

We have the following results for the family of $\mathrm{ABCs} \mathrm{ABC}_{1, T}^{M}$.

Theorem 3. Let $u_{0} \in L^{2}(\Omega)$ an initial data with compact support in $\Omega$. Let $V \in C^{\infty}\left(\mathbb{R}^{2} \times \mathbb{R}^{+}, \mathbb{R}\right)$ be a real-valued potential. We denote by $u$ a solution to the initial boundary value problem

$$
\left\{\begin{array}{l}
i \partial_{t} u+\Delta u+V u=0, \quad \text { in } \Omega_{T}, \\
\partial_{\mathbf{n}} u+\Lambda_{1, T}^{M} u=0, \quad \text { on } \Sigma_{T}, \\
u(x, 0)=u_{0}(x), \quad \text { in } \Omega
\end{array}\right.
$$

where the operators $\Lambda_{1, T}^{M}, M \in\{2,3,4\}$ are defined in Proposition 1. Then, $u$ satisfies the energy inequality

$$
\forall t>0, \quad\|u(t)\|_{L^{2}(\Omega)} \leq\left\|u_{0}\right\|_{L^{2}(\Omega)}
$$

for $M=2$. Furthermore, if $V$ a a radial potential: $V(r, s, t)=V(r, t)$, then the energy inequality (111) is also satisfied for $M=3$ and $M=4$ if we assume that $\operatorname{sg}\left(\partial_{\mathbf{n}} V\right)$ is time independent over $\Sigma$. In particular, this implies the uniqueness of the solution $u$ of the initial boundary value problem (110).

Proof. We first consider the second-order boundary condition for a general potential $V(r, s, t)$. The beginning of the proof is the same as in Theorem 3, until the equality (107). We consider now the boundary condition $\mathrm{ABC}_{1, T}^{2}$ given by the operator (61). We get

$$
\int_{\Sigma \times \mathbb{R}} i \bar{u} \partial_{\mathbf{n}} u d \Sigma d t=-i e^{-i \pi / 4} \int_{\Sigma \times \mathbb{R}} e^{i \mathcal{V}} \bar{u} \partial_{t}^{1 / 2}\left(e^{-i \mathcal{V}} u\right) d \Sigma d t-\frac{i}{2} \int_{\Sigma \times \mathbb{R}} \kappa|u|^{2} d \Sigma d t .
$$

The second term is clearly purely imaginary. The first term can be written as

$$
-i e^{-i \pi / 4} \int_{\Sigma \times \mathbb{R}} e^{i \mathcal{V}_{\bar{u}}} \partial_{t}^{1 / 2}\left(e^{-i \mathcal{V}} u\right) d \Sigma d t=-i e^{-i \pi / 4} \int_{\mathbb{R} \times \mathbb{R}}\left|\mathscr{F}_{(s, t)}\left(e^{-i \mathcal{V}} u\right)(\xi, \tau)\right|^{2} \sqrt{i \tau} d \xi d \tau
$$

and so varies in $\mathbb{R}^{-} \cup i \mathbb{R}^{-}$, which ends the proof for $M=2$. 
Let us assume now that $V=V(r, t)$ is a radial potential. Then the phase function $\mathcal{V}$ is also radial. The operator $\Lambda_{1, T}^{2}$ remains unchanged but since $\partial_{s} \mathcal{V}=0$, the operators $\Lambda_{1, T}^{3}$ and $\Lambda_{1, T}^{4}$ respectively write down

$$
\begin{aligned}
& \Lambda_{1, T}^{3} u=\Lambda_{1, T}^{2} u-e^{i \pi / 4} e^{i \mathcal{V}}\left(\frac{\kappa^{2}}{8}+\frac{\Delta_{\Sigma}}{2}\right) I_{t}^{1 / 2}\left(e^{-i \mathcal{V}} u\right), \\
& \Lambda_{1, T}^{4} u=\Lambda_{1, T}^{3} u+i e^{i \mathcal{V}}\left(\frac{\partial_{s}\left(\kappa \partial_{s}\right)}{2}+\frac{\kappa^{3}+\partial_{s}^{2} \kappa}{8}\right) I_{t}\left(e^{-i \mathcal{V}} u\right)-\frac{i}{4} \operatorname{sg}\left(\partial_{\mathbf{n}} V\right) \sqrt{\left|\partial_{\mathbf{n}} V\right|} e^{i \mathcal{V}} I_{t}\left(\sqrt{\left|\partial_{\mathbf{n}} V\right|} e^{-i \mathcal{V}} u\right),
\end{aligned}
$$

by using the symmetric form of $\Lambda_{1, T}^{4}$. The third order boundary condition considers the additional terms

$$
\begin{aligned}
\int_{\Sigma \times \mathbb{R}} i \bar{u} \partial_{\mathbf{n}} u d \Sigma d t= & \int_{\Sigma \times \mathbb{R}}\left(-i e^{-i \pi / 4} e^{i \mathcal{V}} \bar{u} \partial_{t}^{1 / 2}\left(e^{-i \mathcal{V}} u\right)+i e^{i \pi / 4} e^{i \mathcal{V}} \bar{u} \frac{\Delta_{\Sigma}}{2} I_{t}^{1 / 2}\left(e^{-i \mathcal{V}} u\right)\right) d \Sigma d t \\
& -\frac{i}{2} \int_{\Sigma \times \mathbb{R}} \kappa|u|^{2} d \Sigma d t+i e^{i \pi / 4} \int_{\Sigma \times \mathbb{R}} \frac{\kappa^{2}}{8} e^{i \mathcal{V}} \bar{u} I_{t}^{1 / 2}\left(e^{-i \mathcal{V}} u\right) d \Sigma d t
\end{aligned}
$$

The first term can be treated in a similar way as $\mathrm{ABC}_{2, T}^{M}$ and the second one is purely imaginary. Finally, the third term is analyzed as before. Then, we only obtain some terms with a negative or null real part for the third order ABC. Let us now consider the fourth order ABC for radial potentials. We must study the sign of the additional terms

$$
\begin{aligned}
& -\int_{\Sigma \times \mathbb{R}} \frac{1}{2} e^{i \mathcal{V}} \bar{u} \partial_{s}\left(\kappa \partial_{s}\right) I_{t}\left(e^{-i \mathcal{V}} u\right) d \Sigma d t-\int_{\Sigma \times \mathbb{R}} e^{i \mathcal{V}_{\bar{u}}} \frac{\kappa^{3}+\partial_{s}^{2} \kappa}{8} I_{t}\left(e^{-i \mathcal{V}} u\right) d \Sigma d t \\
& +\int_{\Sigma \times \mathbb{R}} \frac{1}{4} \operatorname{sg}\left(\partial_{\mathbf{n}} V\right) e^{i \mathcal{V}} \sqrt{\left|\partial_{\mathbf{n}} V\right|} \bar{u} I_{t}\left(e^{-i \mathcal{V}} \sqrt{\left|\partial_{\mathbf{n}} V\right|} u\right) d \Sigma d t
\end{aligned}
$$

These three terms can be treated as for $\mathrm{ABC}_{2, T}^{4}$ : the first one by integration by part over $\Sigma$, and next applying the identity (102) after using the assumption over $\operatorname{sg}\left(\partial_{\mathbf{n}} V\right)$ which is time independent. This ends the proof for $M=4$.

Remark 7. Let us remark that the results cannot be extended to $M \geq 3$ when the potential is not radial. Indeed, we then have to study the sign of the real part of the terms

$$
-e^{i \pi / 4} \int_{\mathbb{R} \times \mathbb{R}}\left|\mathscr{F}\left(e^{-i \mathcal{V}} u\right)(\xi, \tau)\right|^{2}\left(\partial_{s} \mathcal{V}\right) \frac{i \xi}{\sqrt{i \tau}} d \xi d \tau
$$

and

$$
i e^{i \pi / 4} \int_{\mathbb{R} \times \mathbb{R}}\left|\mathscr{F}\left(e^{-i \mathcal{V}} u\right)(\xi, \tau)\right|^{2}\left(i \partial_{s}^{2} \mathcal{V}-\left(\partial_{s} \mathcal{V}\right)^{2}\right) \frac{1}{\sqrt{i \tau}} d \xi d \tau
$$

However, the symbol $\left(\partial_{s} \mathcal{V}\right) \frac{i \xi}{\sqrt{i \tau}}$ varies in $e^{i \pi / 4} \mathbb{R} \cup e^{-i \pi / 4} \mathbb{R}$ without restricting the lines to half-lines studies since $\xi$ describes $\mathbb{R}$ and has no fixed sign. Consequently the first integral takes values in $\mathbb{R} \times i \mathbb{R}$ and its real part may be either positive or negative. The same problem arises for the second integral $\left(i \partial_{s}^{2} \mathcal{V}-\left(\partial_{s} \mathcal{V}\right)^{2}\right)$ which is complex. Therefore, estimates can only be obtained for radial potentials for ABCs of order 3 or higher in which case these two integrals vanish. 


\section{Conclusion}

We proposed the construction of absorbing boundary conditions for the two-dimensional Schrödinger equation with a general potential and for a curved convex fictitious boundary. We essentially obtained four families of ABCs according to strategy I (gauge change) or II (direct method), with a Taylor expansion or a Padé approach. The discretization schemes of these ABCs will be introduced and analyzed in a forthcoming paper [6]. Furthermore, numerical examples will show how these ABCs compare.

\section{References}

[1] X. Antoine, A. Arnold, C. Besse, M. Ehrhardt, and A. Schädle. A review of transparent and artificial boundary conditions techniques for linear and nonlinear Schrödinger equations. Commun. Comput. Phys., 4(4):729-796, 2008.

[2] X. Antoine, H. Barucq, and A. Bendali. Bayliss-Turkel-like radiation conditions on surfaces of arbitrary shape. J. Math. Anal. Appl., 229(1):184-211, 1999.

[3] X. Antoine and C. Besse. Construction, structure and asymptotic approximations of a microdifferential transparent boundary condition for the linear Schrödinger equation. J. Math. Pures Appl. (9), 80(7):701-738, 2001.

[4] X. Antoine and C. Besse. Unconditionally stable discretization schemes of non-reflecting boundary conditions for the one-dimensional Schrödinger equation. J. Comput. Phys., 188(1):157-175, 2003.

[5] X. Antoine, C. Besse, and P. Klein. Absorbing boundary conditions for the one-dimensional Schrödinger equation with an exterior repulsive potential. J. Comput. Phys., 228(2):312-335, 2009.

[6] X. Antoine, C. Besse, and P. Klein. Absorbing boundary conditions for the two-dimensional Schrödinger equation with an exterior potential. Part II: Discretization and numerical results. in preparation, 2011.

[7] X. Antoine, C. Besse, and V. Mouysset. Numerical schemes for the simulation of the twodimensional Schrödinger equation using non-reflecting boundary conditions. Math. Comp., 73(248):1779-1799 (electronic), 2004.

[8] X. Antoine, C. Besse, and J. Szeftel. Towards accurate artificial boundary conditions for nonlinear PDEs through examples. Cubo, 11(4):29-48, 2009.

[9] N. Ben Abdallah, F. Méhats, and O. Pinaud. On an open transient Schrödinger-Poisson system. Math. Models Methods Appl. Sci., 15(5):667-688, 2005.

[10] J.-P. Bérenger. A perfectly matched layer for the absorption of electromagnetic waves. J. Comput. Phys., 114(2):185-200, 1994.

[11] A. Boutet de Monvel, A. S. Fokas, and D. Shepelsky. Analysis of the global relation for the nonlinear Schrödinger equation on the half-line. Lett. Math. Phys., 65(3):199-212, 2003. 
[12] R. Carles. Linear vs. nonlinear effects for nonlinear Schrödinger equations with potential. Commun. Contemp. Math., 7(4):483-508, 2005.

[13] T. Cazenave. An introduction to nonlinear Schrödinger equations. Instituto de Matemática-UFRJ, Rio de Janeiro, RJ, 1996.

[14] P. Debernardi and P. Fasano. Quantum confined Stark effect in semiconductor quantum wells including valence band mixing and Coulomb effects. IEEE Journal of Quantum Electronics, 29:2741-2755, 1993.

[15] A. del Campo, G. Garcia-Calderon, and J. G. Muga. Quantum transients. Physics Report-Review Section of Physics Letters, 476(1-3):1-50, May 2009.

[16] B. Engquist and A. Majda. Absorbing boundary conditions for the numerical simulation of waves. Math. Comp., 31(139):629-651, 1977.

[17] B. Engquist and A. Majda. Radiation boundary conditions for acoustic and elastic wave calculations. Comm. Pure Appl. Math., 32(3):313-357, 1979.

[18] R. Lascar. Propagation des singularités des solutions d'équations pseudo-différentielles quasi homogènes. Ann. Inst. Fourier (Grenoble), 27(2):vii-viii, 79-123, 1977.

[19] M. Levy. Non-local boundary conditions for radiowave propagation. Phys. Rev. E, 62(1):13821389, Jul 2000.

[20] M. Levy. Parabolic equation methods for electromagnetic wave propagation, volume 45 of IEE Electromagnetic Waves Series. Institution of Electrical Engineers (IEE), London, 2000.

[21] E. Lorin, A. Bandrauk, and S. Chelkowski. Numerical Maxwell-Schrödinger model for lasermolecule interaction and propagation. Comput. Phys. Comm., 177 (12):908-932, 2007.

[22] E. Lorin, A. Bandrauk, and S. Chelkowski. Mathematical modeling of boundary conditions for laser-molecule time dependent Schrödinger equations and some aspects of their numerical computation-One-dimensional case. Numer. Meth. for P.D.E., 25:110-136, 2009.

[23] J. G. Muga, J. P. Palao, B. Navarro, and I. L. Egusquiza. Complex absorbing potentials. Phys. Rep., 395(6):357-426, 2004.

[24] L. Nirenberg. Pseudodifferential operators and some applications, volume 17 of Regional Conf. Ser. in Math. AMS 17, Lectures on Linear Partial Differential Equations. AMS, 1973.

[25] J. Szeftel. Design of absorbing boundary conditions for Schrödinger equations in $\mathbb{R}^{d}$. SIAM J. Numer. Anal., 42(4):1527-1551 (electronic), 2004.

[26] J. Szeftel. Absorbing boundary conditions for one-dimensional nonlinear Schrödinger equations. Numer. Math., 104(1):103-127, 2006.

[27] O. Vacus. Mathematical analysis of absorbing boundary conditions for the wave equation: The corner problem. Math. Comp., 74(249):177-200, 2004. 
[28] C. Zheng. A perfectly matched layer approach to the nonlinear Schrödinger wave equations. $J$. Comput. Phys., 227(1):537-556, 2007.

[29] C. Zheng. An exact absorbing boundary condition for the Schrödinger equation with sinusoidal potentials at infinity. Commun. Comput. Phys., 3(3):641-658, 2008. 